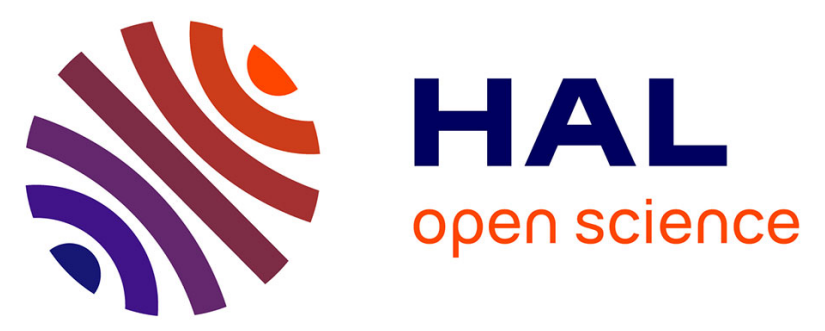

\title{
La céramique gallo-romaine du Bas-Empire à Arras (Nemetacum), Pas-de-Calais
}

\author{
Marie Tuffreau-Libre, Alain Jacques
}

\section{To cite this version:}

Marie Tuffreau-Libre, Alain Jacques. La céramique gallo-romaine du Bas-Empire à Arras (Nemetacum), Pas-de-Calais. Gallia - Fouilles et monuments archéologiques en France métropolitaine, 1992, 49, pp.99-127. 10.3406/galia.1992.2932 . hal-01915250

\section{HAL Id: hal-01915250 \\ https://hal.science/hal-01915250}

Submitted on 19 Jan 2020

HAL is a multi-disciplinary open access archive for the deposit and dissemination of scientific research documents, whether they are published or not. The documents may come from teaching and research institutions in France or abroad, or from public or private research centers.
L'archive ouverte pluridisciplinaire HAL, est destinée au dépôt et à la diffusion de documents scientifiques de niveau recherche, publiés ou non, émanant des établissements d'enseignement et de recherche français ou étrangers, des laboratoires publics ou privés.

\section{(ㅇ)(1) $\$$}

Distributed under a Creative Commons Attribution - NonCommercial - NoDerivatives $\mid 4.0$ 


\title{
La céramique gallo-romaine du Bas-Empire à Arras (Nemetacum) (Pas-de-Calais)
}

\author{
par Marie TUFFREAU-LIBRE* et Alain JACQUES **
}

Les découvertes récentes d'Arras ont mis au jour d'importantes séries de céramiques gallo-romaines - Sdives qui ont permis d'esquisser une évolution de la céramique du Bas-Empire. Cette étude vient préciser les daijos d'apparition des formes traditionnelles, ce qui modifie la vision générale du IV ${ }^{\mathrm{e}} \mathbf{s}$. puisque cette période ạparaît finalement bien représentée dans les villes du Nord de la Gaule. L'étude des céramiques fines et des productions particulières (cérarnique de l'Eifel, céramique modelée) conduit à mieux apprécier le volume des importations ainsi qu'à mettre en évidence la présence à Arras à la fin du Bas-Empire d'un groupe de population étranger qu'illustre le mobilier trouvé dans le sanctuaire germanique.

Recent discoveries in Arras (Pas-de-Calais) permitted to consider important series of gallo-roman potteries from the Lower-Empire. Their study brings many informations for that period in the north of Gaul. Hand-made potteries show the presence, on the site, in the end of the IVth century, of German people.

Mots clés : céramique, Bas-Empire, typologie, nécropole, ville, datation, poterie, sanctuaire, comptage, Arras, Pas-de-Calais.

* C.NRS, UMR 126, 12, rue de Bailleulval, Berles-aux-Bois, 62123 Beaumetz-les-Loges.

** Service archéologique, Palais Saint-Vaast, 62000 Arras. 
La céramique du Bas-Empire est demeurée jusqu'à ce jour assez mal connue. Sa chronologie reste encore floue, comparée à celle des répertoires du $\mathrm{I}^{\mathrm{er}} \mathrm{s}$. dont l'évolution rapide fournit des repères plus précis. Cet état de chose tient à deux faits. Tout d'abord, il faut reconnaître que la céramique sigillée nous a longtemps induit en erreur. Datée par rapport à cette dernière, la céramique commune du ${ }_{1 I}{ }^{\mathrm{e}} \mathrm{s}$. $\mathrm{s}^{\prime}$ est vue souvent rejetée au II $^{\mathrm{e}} \mathrm{s}$. De même, la rareté de la céramique du $v^{e} s$. s'explique surtout par des décalages similaires. La plupart des formes de cette période ont été classées dans le $\mathrm{IV}^{\mathrm{e}} \mathrm{s}$. en fonction de la céramique sigillée d Argonne, dont la datation se voit régulièrement reconsidérée. L'origine de ces erreurs réside aussi dans une utilisation parfois trop systématique des datations fournies par la numismatique. Enfin, beaucoup de chronotypologies ont été établies à partir d'objets issus des fouilles de nécropoles, alors que la disparité de ces ensembles les rend peu fiables pour ce genre d'étude.

Ces problèmes sont apparus nettement lors des fouilles récentes menées à Arras (Pas-de-Calais) (fig. 1), où les trouvailles monétaires ne correspondaient pas aux datations proposées pour la céramique par la bibliographie ancienne. De nombreuses formes communes datées précédemment avec de la sigillée et des échantillonnages réduits de monnaies sont apparus plus tardifs, avec des différences allant parfois jusqu'à un siècle.

Tous ces éléments nouveaux nous ont conduit à reconsidérer l'ensemble de la céramique du BasEmpire dans le Nord et le Pas-de-Calais. Dans cette optique, nous présentons ici une première synthèse concernant la céramique tardive trouvée à Arras. Les objets étudiés proviennent des fouilles de la Préfecture, menées de 1977 à 1980 (Jacques et alii, 1983), et surtout de celles du site de Baudimont, exploré depuis 1984 (Les cultes à Arras au BasEmpire, 1990) (fig. 2). Cette dernière fouille, d'une superficie de $4000 \mathrm{~m}^{2}$ a permis des constatations importantes sur l'urbanisme de la ville. Elle a surtout été l'occasion de découvertes exceptionnelles. L'édifice cultuel dédié aux dieux orientaux Attis et Cybèle, découvert en 1986, est la première manifestation d'une parure monumentale jusqu'à présent inconnue à Nemetacum. La mise en évidence de structures correspondant à un sanctuaire germanique éclaire d'un jour nouveau la germanisation de la Gaule du Nord. Les casernes théodosiennes sont l'indice d'une présence militaire à Arras jusqu'au début $d u v^{e} s$. Les datations de ces ensembles sont fournies par la numismatique (Gricourt, 1987) : vers le milieu du $I^{e} \mathbf{s}$. pour le site de la Préfecture,

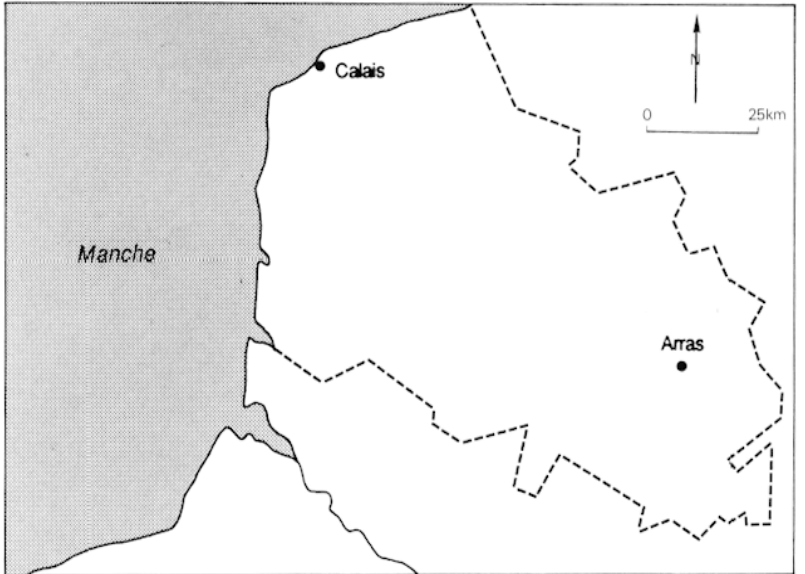

Fig. 1 - Situation d'Arras (Pas-de-Calais) dans le .Vord de la France.

seconde moitié du $\operatorname{III}^{\mathrm{e}} \mathrm{s}$. pour la construction du temple d'Attis-Cybèle, vers 375 pour les remblais correspondant à la destruction de ce même sanctuaire, de 380 au premier quart du ves. pour les deux niveaux d'occupation des casernes.

Cette étude apporte de nombreux renseignements sur la chronologie de la céramique des II $^{\mathrm{e}}$ et IV ${ }^{\mathrm{e}}$ s., fondée sur des structures d'habitats aux datations fiables. Du point de vue typologique, peu d'éléments nouveaux apparaissent car les répertoires du Nord de la France (Nord et Pas-de-Calais) commencent à être bien connus. En plus des éléments concernant les domaines économiques et religieux, les trouvailles d'Arras sont aussi l'occasion de soulever certains problèmes méthodologiques. Nous ne prétendons pas apporter ici de réponses définitives. Il s'agit d'une mise au point des connaissances actuelles.

\section{ASPECTS GÉNÉRAUX DE LA CÉRAMIQUE DU BAS-EMPIRE À ARRAS}

\section{L'ÉVOLUTION DES RÉPERTOIRES}

Les résultats obtenus ont mis en évidence un fait important dans l'étude de la céramique : les répertoires mis en place à la fin du $\mathrm{I}^{\mathrm{er}} \mathrm{s}$. et au $\mathrm{II}^{\mathrm{e}} \mathrm{s}$. semblent en effet durer très longtemps sans changement. Certains types disparaissent, laissant la place à d'autres fabrications, mais la majeure partie des formes ne varient pas. Par contre, leurs pourcentages peuvent être très différents, et il est possible d'affiner la chronologie en utilisant des comptages, afin de suivre la naissance, l'apogée et le déclin des formes en céramique commune. Nous avons donc essayé de 


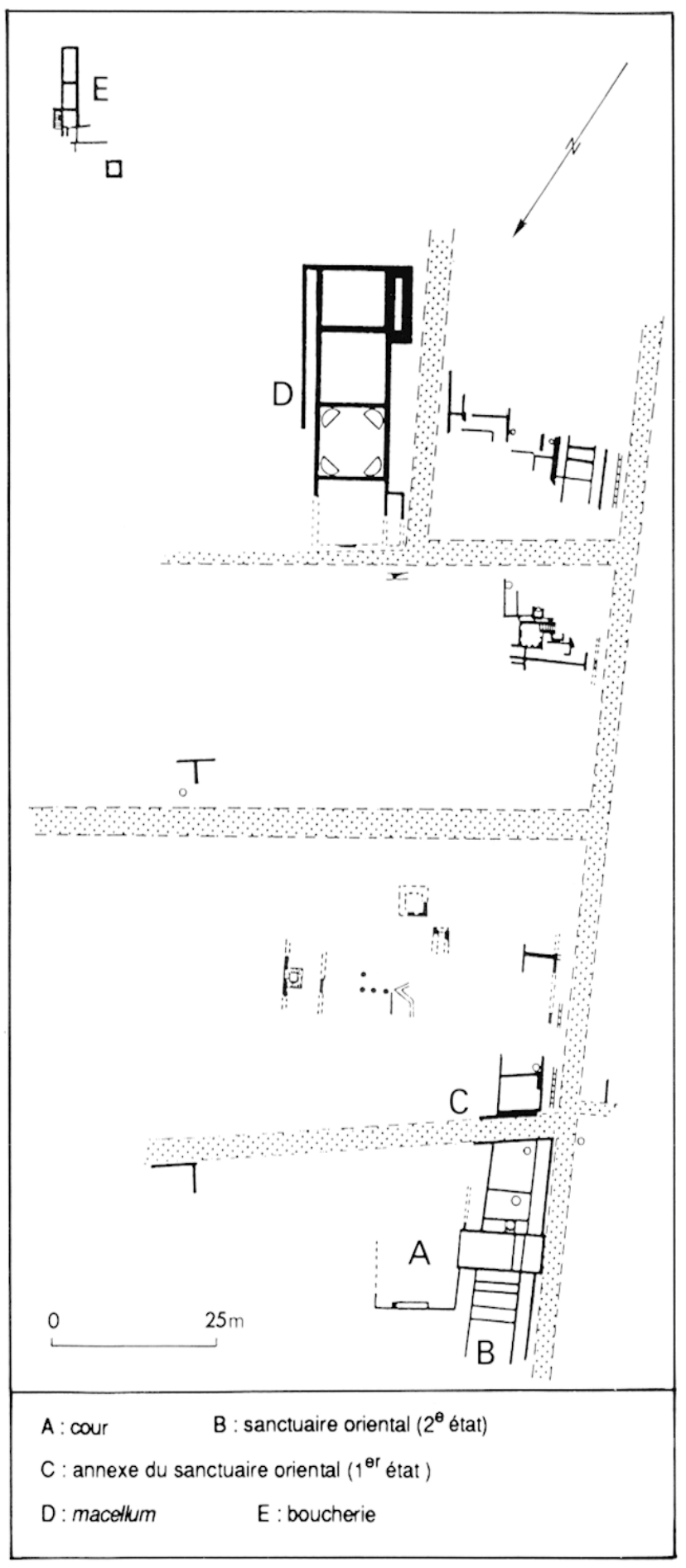

Fig. 2 - Plan général des fouilles de Baudimont.

retracer l'évolution de la céramique d'Arras dans un tableau indiquant pour chaque époque les formes existantes et leur fréquence en pourcentage, les comptages ayant été réalisés sur les formes complètes et les bords des céramiques typologiquement identifiables (nombre minimum d'individus : N.M.I.) (fig. 3, tabl. I).
Cette étude repose sur les ensembles comparés de la Préfecture, des couches de comblement du sanctuaire d'Attis-Cybèle (premier et deuxième état) et de celles des deux phases d'occupation des casernes. Les caractères homogènes des structures (remblais, dépotoirs) rendent les comparaisons d'autant plus signifiantes. La céramique du sanctuaire germanique apporte surtout des renseignements dans un autre domaine et sera donc traitée à part.

\section{LA CÉRAMIQUE DE LA SECONDE MOITIÉ DU III ${ }^{\mathrm{e}} \mathrm{S}$.}

Le sanctuaire d'Attis et Cybèle semble avoir été construit vers le milieu du III $^{\mathbf{e}} \mathrm{s}$. Aucune monnaie n'a été découverte dans les niveaux contemporains de cette édification. Par contre, la céramique trouvée dans les remblais est identique dans sa totalité à celle recueillie dans un puits daté après 257 par les monnaies, ce qui nous permet de situer ces deux ensembles dans la seconde moitié du II $^{\mathrm{e}} \mathrm{s}$.

Les assiettes carénées de type Ila (fig. 4, n ${ }^{\circ 5} 5$ et 6) sont alors bien représentées (fig. 3). Cette forme appartient au répertoire mis en place à la fin du $\mathrm{i}^{\mathrm{er}} \mathrm{s}$. (Tuffreau-Libre, 1980a). Très abondante au $\mathrm{Ir}^{\mathrm{e}} \mathrm{s}$., elle voit sa représentation baisser lentement au cours des $\mathrm{III}^{\mathrm{e}}$ et $\mathrm{IV}^{\mathrm{e}} \mathrm{s}$., pour disparaître à la fin du $\mathrm{IV}^{\mathrm{e}} \mathrm{s}$., où elle n'apparaît plus que de façon résiduelle. Il s'agit d'un type répandu sur les sites du Nord, du Pas-deCalais et de la Somme (Bayard, 1980).

Les assiettes à enduit rouge pompéien (fig. 4, $\left.\mathrm{n}^{\mathrm{os}} 2,3,4\right)$ sont également nombreuses dans cette couche. La datation de cette forme a longtemps été limitée du $\mathrm{I}^{\mathrm{er}} \mathrm{s}$. au début $\mathrm{du} \mathrm{III}^{\mathrm{e}} \mathrm{s}$. En fait, des fouilles récentes sur des sites comme Graincourt-lesHavrincourt (Machut, Tuffreau-Libre, 1991), Famars (Vanbrugghe, 1989) et Arras montrent l'importance quantitative de ces assiettes aux III $^{\mathrm{e}}$ et $\mathrm{IV}^{\mathrm{e}} \mathrm{s}$., ce qui exclut la possibilité d'une céramique de caractère résiduel. Ce type est bien connu sur les sites du Nord, du Pas-de-Calais et de la Belgique actuelle.

Les bols à panse ronde, à lèvre allongée et repliée paraissent représentatifs du $\mathrm{II}^{\mathrm{e}} \mathrm{s}$. (type $\mathrm{IVb}$, fig. $4, n^{\circ} 8$ ). Ils sont par exemple très courants sur le site de Famars, un des lieux de production connu. Les bols carénés de ce remblai se rattachent encore aux productions connues dès la fin du $\mathrm{I}^{\mathrm{er}} \mathrm{s}$. et durant tout le II $^{\mathrm{e}} \mathrm{s}$. : bol caréné XVIa, XVIc, bol caréné décoré de bandes lissées entrecroisées (fig. $5, \mathrm{n}^{\text {os }} 1,2$ ).

De même, les marmites à panse aplatie, décorées de bandes lissées entrecroisées (fig. $5, \mathrm{n}^{\circ} 12$ ) sont encore en nombre important. Cette forme, apparue dès la fin du $\mathrm{I}^{\mathrm{er}} \mathrm{s}$., est fréquente au $\mathrm{II}^{\mathrm{e}} \mathrm{s}$. et accompagne généralement les bols décorés de façon 


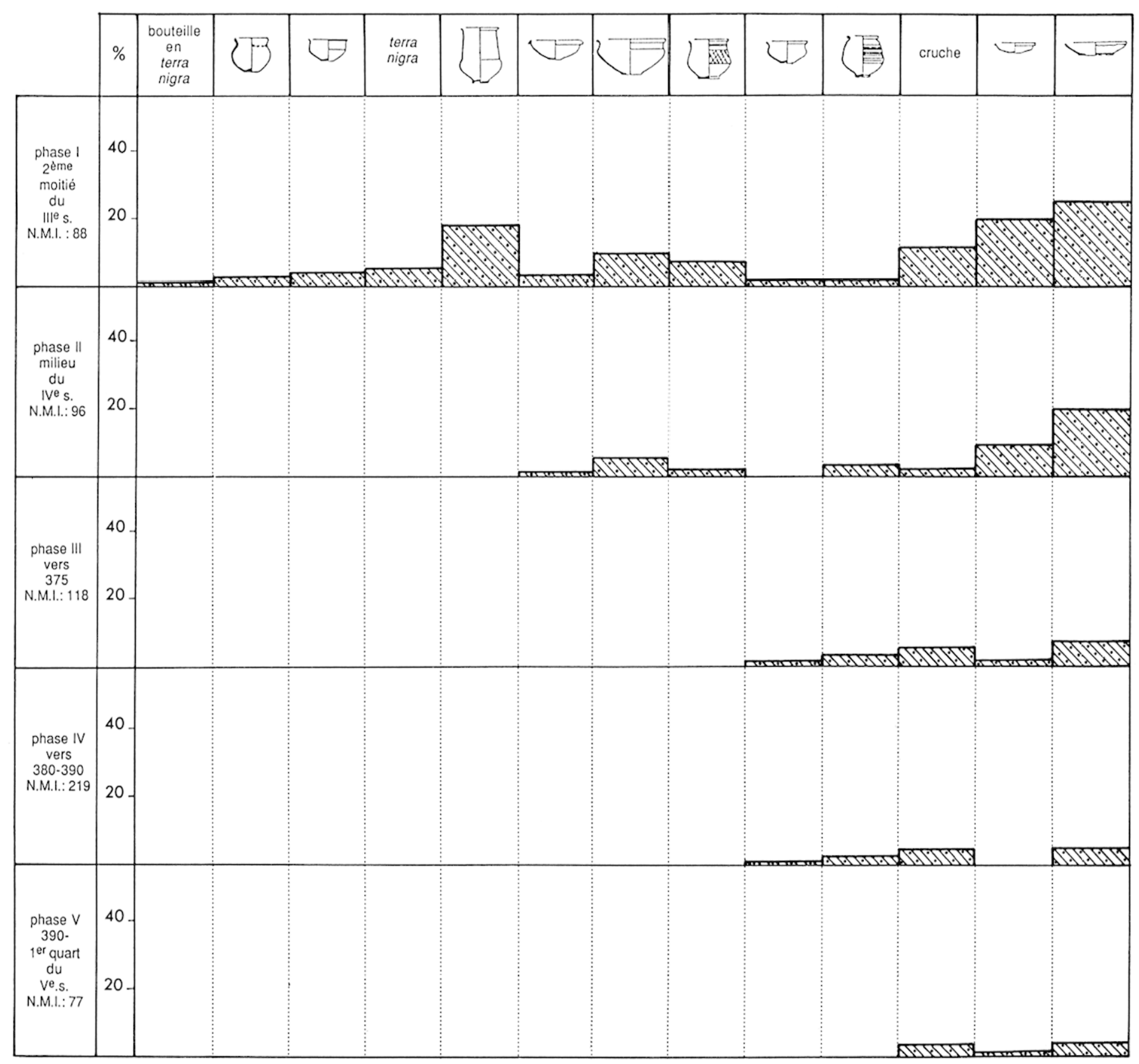

Fig. 3 - Tableau illustrant la répartition de la céramique à Arras, de la fin du $\operatorname{III}^{\mathrm{e}} \mathrm{s}$. au début du ve $\mathrm{s}$. 


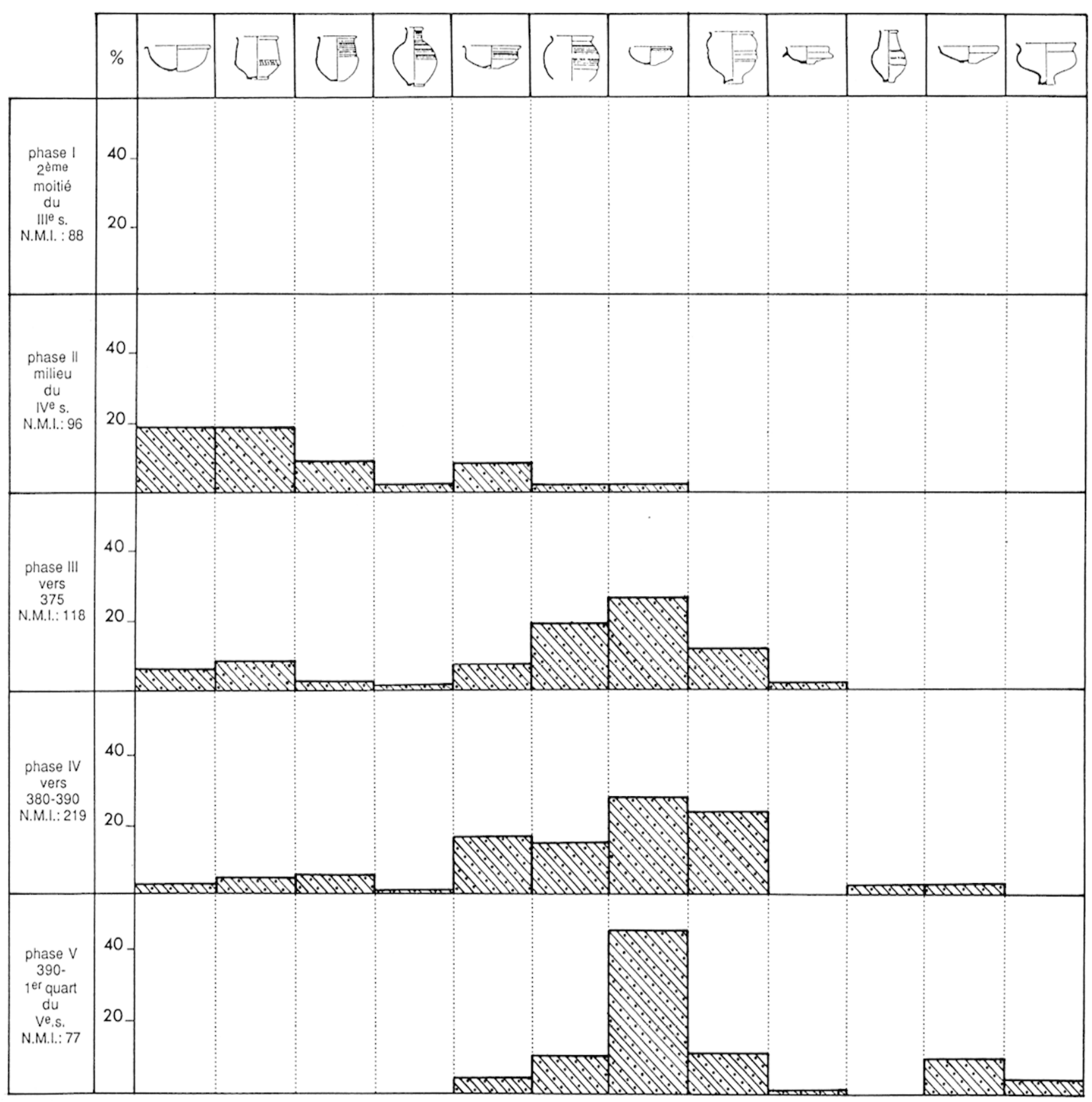


Tableau I - Proportion des différents types de céramique à Arras de la fin du $1 \mathbf{I}^{\mathrm{e}} \mathrm{s}$. au début du $\mathrm{v}^{\mathrm{e}} \mathrm{s}$. (en pourcentages).

\begin{tabular}{|c|c|c|c|c|c|}
\hline PÉRIODE & Phase I & Phase II & Phase III & Phase IV & Phase $V$ \\
\hline ass. PO IIIa $\ldots \ldots \ldots \ldots \ldots$ & 23 & 19 & 6 & 4 & 3 \\
\hline ass. CIIa $\ldots \ldots \ldots \ldots \ldots \ldots$ & 18 & 8 & 2 & - & 1 \\
\hline$\ldots \ldots \ldots \ldots \ldots$ & - & 2 & 25 & 25 & 43 \\
\hline bol RIVb $\ldots \ldots \ldots \ldots \ldots \ldots$ & 2 & 1 & - & - & - \\
\hline bol $\mathrm{RVa} \ldots \ldots \ldots \ldots \ldots$ & - & 17 & 5 & 2 & - \\
\hline bol RXIIIa ............... & - & - & 1 & - & - \\
\hline bol CIIb ............... & 3 & - & - & - & - \\
\hline bol CVIIIa $\ldots \ldots \ldots \ldots \ldots$ & - & - & - & 2 & 10 \\
\hline bol CXIc $\ldots \ldots \ldots \ldots \ldots$ & - & 2 & 7 & 14 & 4 \\
\hline bol CXVIa $\ldots \ldots \ldots \ldots \ldots$ & 8 & 5 & - & - & - \\
\hline bol CXVIc $\ldots \ldots \ldots \ldots \ldots$ & 1 & - & 2 & 1 & - \\
\hline bol collerette $\ldots \ldots \ldots \ldots \ldots$ & - & - & 2 & - & 1 \\
\hline marmite PAIIa $\ldots \ldots \ldots \ldots$ & 6 & 2 & 2 & - & - \\
\hline marmite $\mathrm{PA} \ldots \ldots \ldots \ldots$ & - & 17 & 7 & 4 & - \\
\hline vase PRIVa $\ldots \ldots \ldots \ldots \ldots$ & 2 & - & - & - & - \\
\hline vase PRXIa $\ldots \ldots \ldots \ldots \ldots$ & - & 2 & 17 & 12 & 10 \\
\hline vase PAIb $\ldots \ldots \ldots \ldots \ldots$ & 1 & 3 & 3 & 2 & - \\
\hline vase tronc. $\ldots \ldots \ldots \ldots \ldots$ & 16 & 7 & 3 & 5 & - \\
\hline vase bilobé $\ldots \ldots \ldots \ldots \ldots$ & - & - & 11 & 22 & 12 \\
\hline vase $\mathrm{Ch} .342 \ldots \ldots \ldots \ldots$ & - & - & - & - & 3 \\
\hline vase caréné $\ldots \ldots \ldots \ldots \ldots$. & - & - & - & - & 1 \\
\hline bouteille .................. & - & 2 & 1 & 1 & - \\
\hline bouteille tronc. ............. & - & - & - & 2 & - \\
\hline bout. terra nigra ............ & 1 & - & - & - & - \\
\hline cruche $\ldots \ldots \ldots \ldots \ldots \ldots$ & 11 & 2 & 5 & 4 & 3 \\
\hline terra nigra $1 \mathrm{~s} . \ldots \ldots \ldots \ldots$ & 5 & - & - & - & - \\
\hline non tournée $\ldots \ldots \ldots \ldots \ldots \ldots$ & 2 & - & 1 & 3 & 1 \\
\hline granuleuse $\ldots \ldots \ldots \ldots \ldots$ & - & - & 1 & 1 & 8 \\
\hline
\end{tabular}

identique. Les lignes entrecroisées sont peu à peu remplacées au cours du ${ }^{\prime \prime I}{ }^{e}$ s. par des bandes parallèles groupées en deux ou trois séries. La forme se modifie et devient plus haute (fig. $5, \mathrm{n}^{\circ} 11$ ).

Les vases tronconiques constituent la forme la plus représentative des répertoires du Nord, du Pasde-Calais et de la Picardie. Il faut noter aussi la présence de fragments de vases au décor caractéristique de carrés réalisés à la molette, bien connus dans l'officine de Labuissière, signalés aussi sur le site du III $^{\mathrm{e}} \mathrm{s}$. de Famars. Ces productions ont pu exister dès le II $^{\mathrm{e}} \mathrm{s}$.

Ces objets appartiennent donc au répertoire régional du Haut-Empire, mis en place à la fin du $\mathrm{I}^{\mathrm{er}} \mathrm{s}$. (Tuffreau-Libre, 1988). La disparition des formes résiduelles du $I^{\text {er }} \mathrm{s}$., la rareté des tessons en lerra nigra, l'apparition des premiers types annonçant les périodes plus tardives comme les vases à panse aplatie (type Ib, fig. $6, n^{\circ} 1$ ) qui se substituent progressivement aux marmites à décor entrecroisé, tous ces éléments indiquent une datation dans le III $^{\mathrm{e}} \mathrm{s}$. Le diagramme de cette série se rapproche fortement de celui de Lewarde II (fin du III ${ }^{\mathrm{e}}$, début du IV s.) (Jacques et alii, 1983).

\section{L'ÉVOLUTION DU RÉPERTOIRE AU IV' $\mathrm{S}$.}

Une phase nous manque, celle correspondant au début du $1 v^{e} s$. Un ensemble récemment mis au jour durant l'été 1990 vient combler cette lacune, mais il n'a pu encore être exploité complètement. Les premiers résultats indiquent notamment l'apparition à cette époque des productions de vases tronconiques et moulurés en lerra nigra tardive, très répandus ensuite sur le site de la Préfecture, daté du milieu du IV $\mathrm{s}$.

Les séries de céramiques provenant de cette dernière fouille montrent une diminution des profils qui caractérisaient la période précédente (fig. 3 , phase II) : les assiettes carénées Ila et les assiettes à enduit rouge pompéien sont en régression, ainsi que les formes traditionnelles de bols carénés (type XVIa, XVIc), remplacés par des types un peu différents (bols carénés XIc et XIVa, fig. $5, n^{\circ \mathrm{s}} 3$ à 6 ). Les marmites à panse aplatie IIa n'ont plus qu'une proportion résiduelle et les vases tronconiques sont aussi en diminution très nette. Dans cette dernière catégorie, c'est la variante tardive, à panse plus aplatie qui domine (fig. 6, $\mathrm{n}^{\circ} 9$ ). Certains types ont 

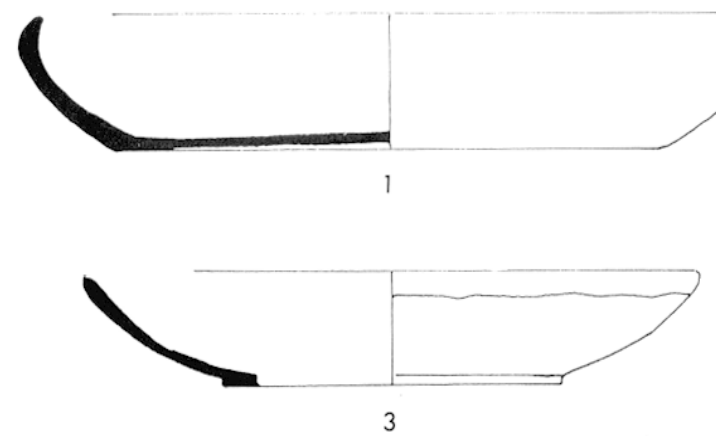
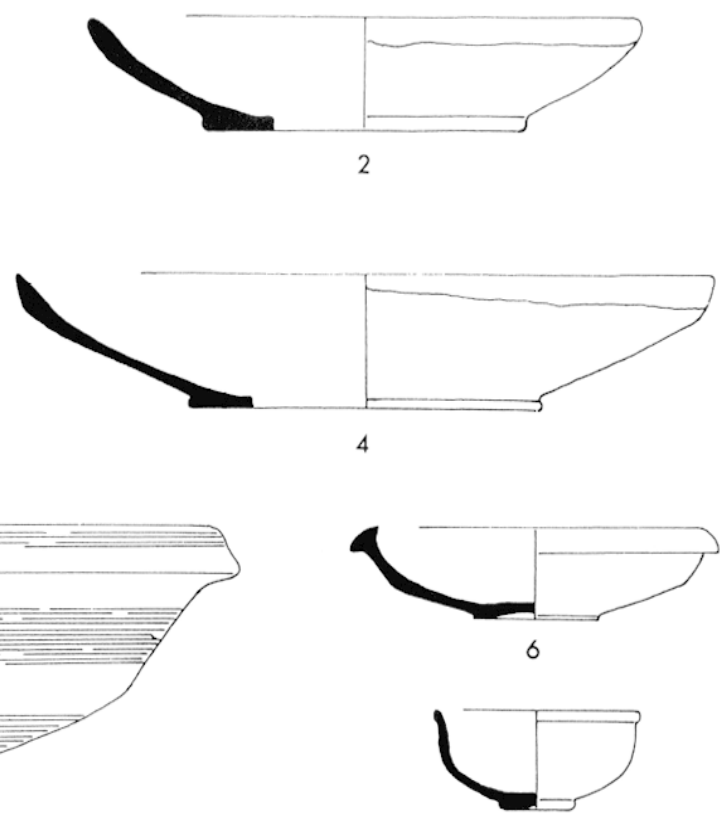

7

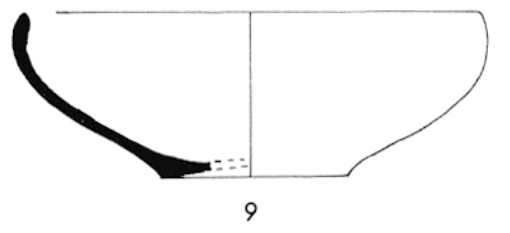

8

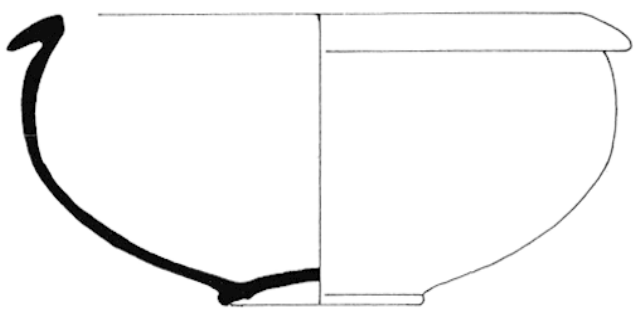

10

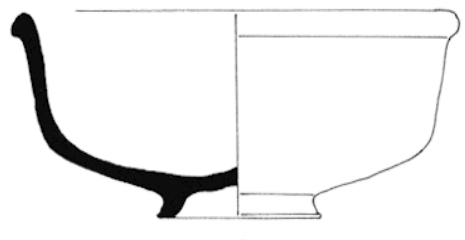

12
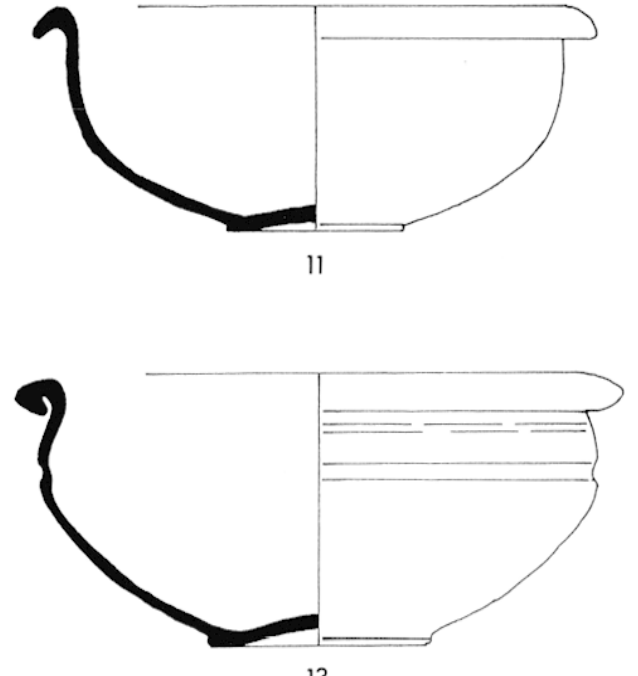

Fig. 4 - Les formes de céramique communes et fines connues à Arras.

complètement disparu, comme le bol rond IVb, remplacé par le bol rond $\mathrm{Va}$ (fig. $4, \mathrm{n}^{\mathrm{os}} 10,11$ ).

Par contre, des types nouveaux apparaissent. C'est le cas du bol rond Ia (fig. $4, \mathrm{n}^{\circ} 9$ ) encore peu fabriqué et, semble-t-il, tout au début de sa diffusion. Il n'est pas encore présent dans les niveaux du début du Ive s. Le bol rond XIIIa est connu à cette période mais sa représentation sur le site demeure faible de façon générale (fig. $4, \mathrm{n}^{\circ} 7$ ).

Une des formes nouvelles est par contre fréquente sur le site. Il s'agit de marmites dérivées à la fois des marmites à panse aplatie IIa et des vases 

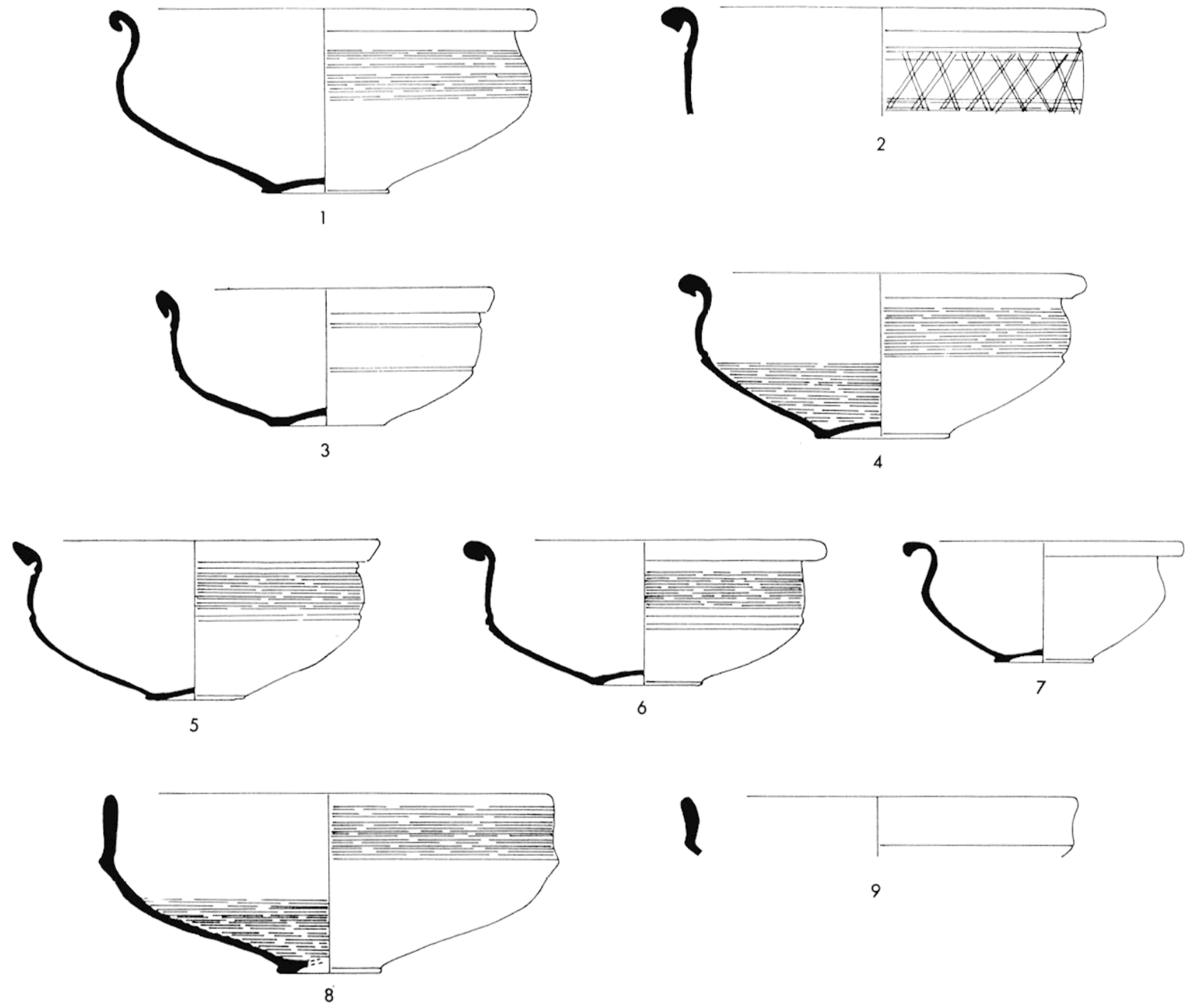

1

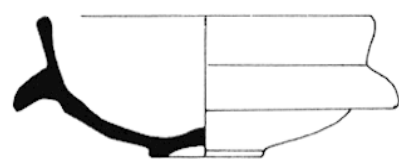

10

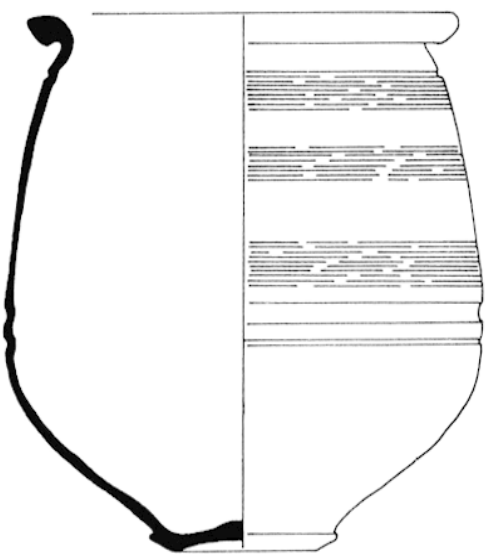

11

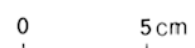

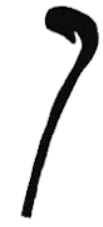

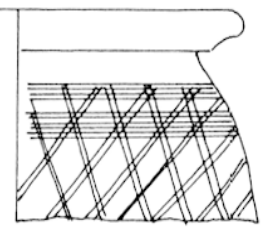

12

Fig. 5 - Les formes de céramiques communes et fines connues à Arras. 

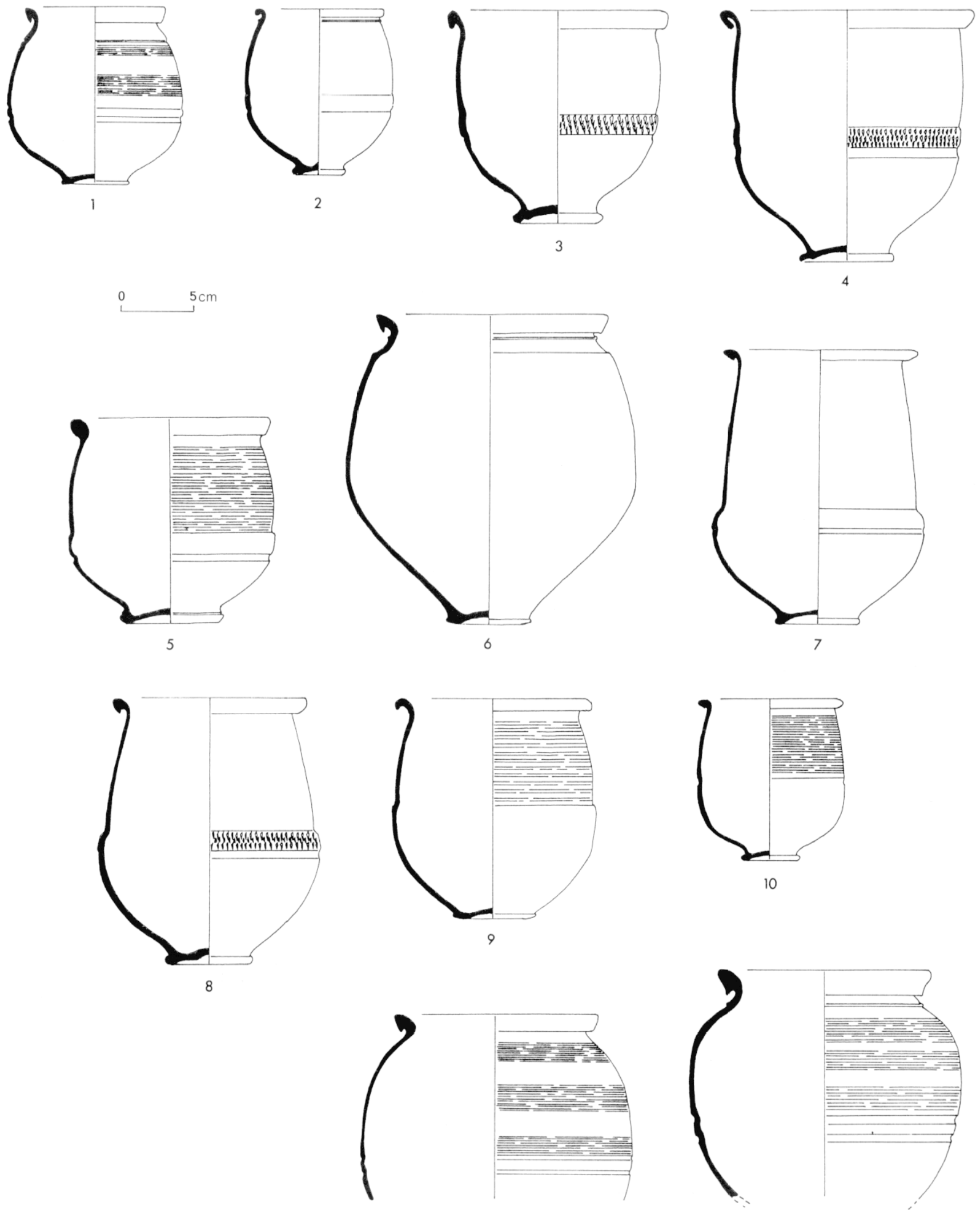

Fig. 6 - Les formes de céramiques communes et fines connues à Arras. 
tronconiques, caractérisées par une moulure centrale souvent décorée de guillochis (fig. $6, \mathrm{n}^{\mathrm{os}} 3,4,5$ ). Le col est décoré de bandes lissées parallèles en séries régulières ou discontinues, en groupes de trois ou encore de séries de guillochis parallèles. Ces fabrications sont souvent réalisées en pâte fine de type terra nigra tardive. Les vases à panse ronde (type XIa, fig. $6, n^{0 s} 11,12$ ), décorés de séries de bandes lissées, connaissent le début de leur diffusion et se substituent progressivement au type Ib des vases à panse aplatie. Les bouteilles à bandes lissées parallèles caractérisent également cette période mais gardent une faible représentation, même par la suite (fig. 7, $\left.\mathrm{n}^{\circ} 11\right)$. On signale pour la première fois la présence de cruches tréflées de style tardif (fig. 7, ${ }^{0} 13$ ).

L'évolution se poursuit à la fin du troisième quart du IV $^{\mathrm{e}} \mathrm{s}$. (comblement du sanctuaire d'AttisCybèle) : augmentation des formes apparues précédemment (bol rond la, vase rond XIa), diminution des types résiduels (fig. 3 , phase III). Les bols ronds imités du type 320 de Chenet (Chenet, 1941) (fig. 4, $\mathrm{n}^{\mathrm{o}} 12$ ), le bol à collerette (fig. $5, \mathrm{n}^{\circ} 10$ ), et surtout le vase bilobé mouluré constituent des nouveautés (fig. $7, n^{\circ} 1$ ).

Cette dernière forme augmente considérablement dans le dernier quart du $\mathrm{IV}^{\mathrm{e}} \mathrm{s}$. (première phase d'occupation des casernes, environ 380 à 390), pour devenir avec le bol rond Ia et le vase rond Xla un des types les plus fréquents et les plus représentatifs de la fin du IV s. (fig. 3, phase IV). A cette époque apparaissent pour la première fois les bols carénés de type VIIIa et leurs variantes (fig. $5, n^{0 s} 8,9$ ), les bouteilles tronconiques (fig. $7, \mathrm{n}^{\circ} 12$ ). Un exemplaire unique de vase, retrouvé dans ce niveau et réalisé en terra nigra, peut se rattacher aux productions dérivées du type 341 de Chenet, fréquentes dans les nécropoles tardives de la forêt de Compiègne (Tuffreau-Libre, 1977) et de la Marne (Collections du M.A.N. de Saint-Germain-en-Laye) (fig. 7, no 7).

La dernière période (deuxième phase d'occupation des casernes et abandon, de 390 environ au premier quart du ve s.) semble enregistrer une simplification $\mathrm{du}$ répertoire dont les variantes deviennent moins nombreuses (fig. 3, phase V). Ce n'est que dans ce niveau qu'on signale les vases à piédestal issus du type 342 de Chenet (fig. $7, n^{\circ} 8$ ), ainsi que les vases carénés, encore une fois bien connus dans les sépultures de la forêt de Compiègne et de la Marne (fig. 7, $\left.n^{\circ} 6\right)$. Les vases bilobés moulurés commencent alors à diminuer au profit d'une forme dérivée, plus simple, très répandue dans toutes les sépultures tardives, mais qui n'est présente ici que par un seul exemplaire, découvert dans le niveau le plus tardif (fin du premier quart du $v^{\mathrm{e}}$ s.) (fig. $7, \mathrm{n}^{\mathrm{o}} 2$ ).
Les fouilles d'Arras permettent donc de préciser nettement la date d'apparition des formes du ive $s$. et notamment de celles considérées traditionnellement comme représentatives du Bas-Empire, en les situant d'ailleurs très tard. Les bouteilles tronconiques ne sont pas répertoriées à Arras avant le dernier quart du $\mathrm{IV}^{\mathrm{e}} \mathrm{s}$. Le vase bilobé de type tardif, les dérivés des vases Chenet 342 voient par exemple - leur première datation repoussée dans le premier quart du $\mathrm{v}^{\mathrm{e}} \mathrm{s}$. et même probablement plutôt à la fin de cette période. Notons l'absence des bols à collerette de type tardif dérivés de Chenet 324 et des imitations en pâte blanche des cruches peintes de l'Argonne, types pourtant présents dans les nécropoles théoriquement contemporaines.

D'autre part, l'évolution que nous venons de retracer montre bien la lenteur de transformations des répertoires, ainsi que l'importance de la céramique de type résiduel, pour autant qu'on puisse la considérer comme telle, en l'absence de renseignements suffisants concernant la durée de fabrication des formes dans les officines de céramique commune. Globalement, le répertoire des céramiques communes change relativement peu $d u{ }_{i I^{e}}$ à la fin $d u$ IV $^{\mathrm{e}} \mathrm{s}$. Il faut souligner aussi des différences dans la représentation des types. Certains d'entre eux sont fabriqués à de nombreux exemplaires, et durant de longues périodes. Il s'agit souvent des objets les plus représentatifs des séries régionales. D'autres, au contraire, ont une durée de vie très courte ou n'apparaissent qu'en nombre toujours très limité, ce qui peut s'expliquer par la présence d'objets d'utilisation moins courante ou représentatifs d'importations de régions voisines.

Ces considérations mettent en évidence un des aspects méthodologiques de la datation de la céramique. Seuls doivent être pris en compte des ensembles, et non des objets isolés, analysés sous des aspects typologiques et quantitatifs. En effet, en l'absence de comptage, il n'est pas possible d'apprécier la réalité des séries, ce qui conduit à des erreurs chronologiques. Actuellement, ces pourcentages sont réalisés sur des catégories typologiques élargies. II est possible d'envisager par la suite des analyses plus poussées encore, à partir des variantes de chaque forme. Cette méthode a évidemment ses limites, et on ne saurait prétendre résoudre ainsi tous les problèmes. Les résultats ne sont pas parfaits, d'autant plus que la représentation de certains types, plus facilement identifiables que d'autres, peut être surévaluée. Cependant, ces images, toutes approximatives qu'elles soient, permettent une meilleure approche de la réalité. 


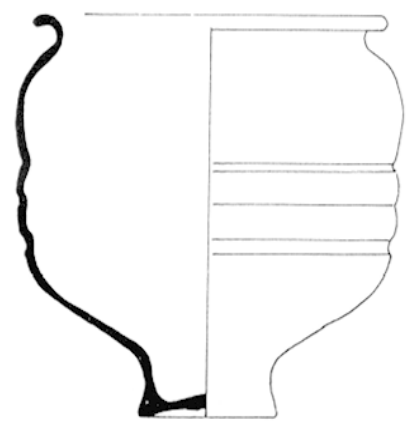

1
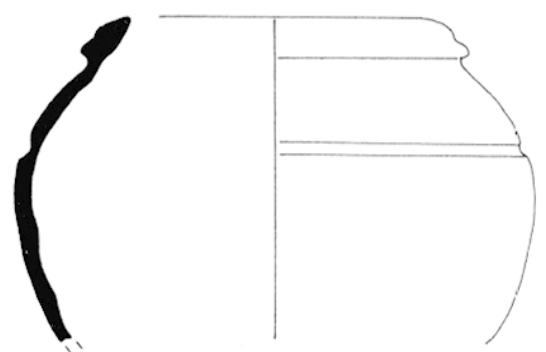

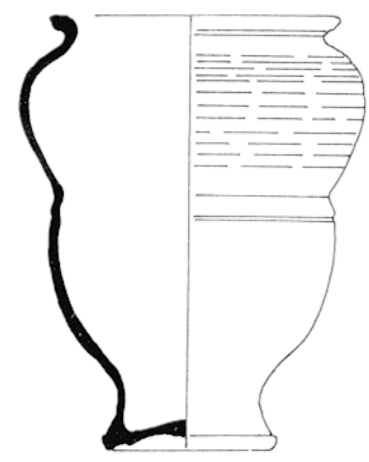

2

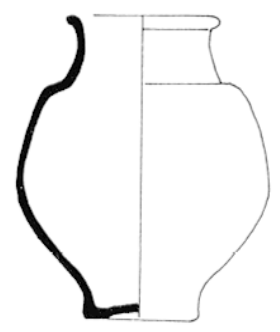

5

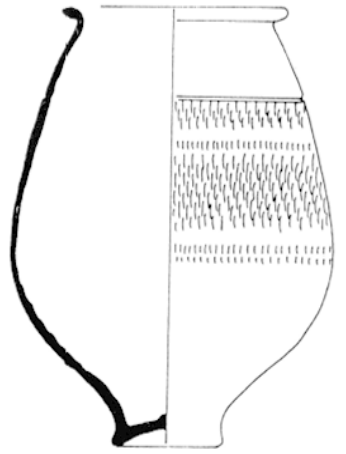

3

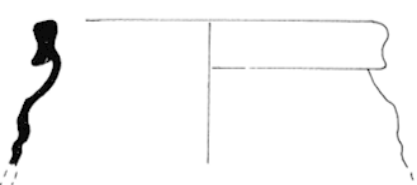

6

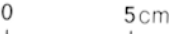

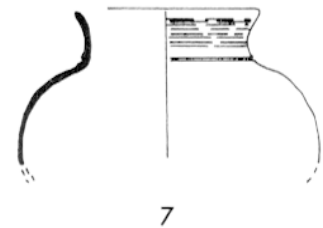

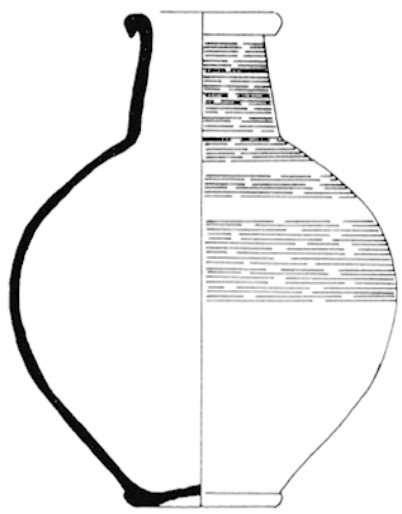

11

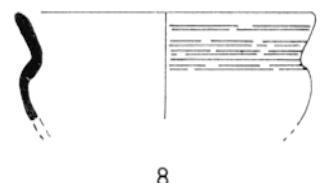

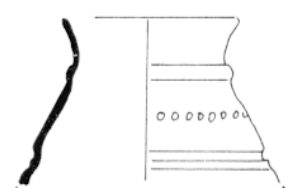

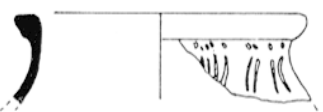

10

Fig. 7 - Les formes de céramiques communes et fines connues à Arras.

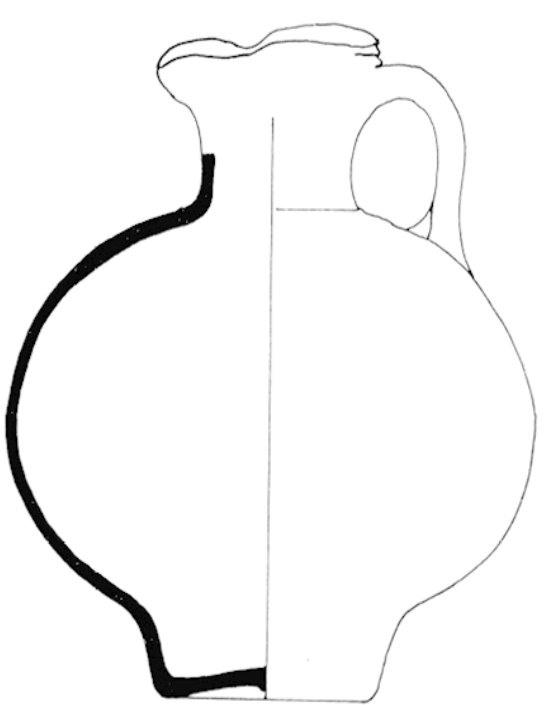

13
Enfin, ces nouveaux éléments modifient de nombreuses datations régionales. Des ensembles provenant de fouilles anciennes effectuées à Arras doivent désormais être datés dans le $I v^{e} s$. et non au
III ${ }^{\mathrm{C}}$ s. (Jelski, 1980). Il en est de même pour ceux d'Amiens qui présentent de nombreuses analogies avec les séries de la seconde moitié du $\mathbf{I V}^{\mathbf{e}} \mathbf{s}$. d'Arras (Bayard, 1980). Les datations avancées à Bavai 
(Nord) semblent trop axées sur le ${ }_{11}{ }^{\mathrm{e}}$ s. et il est probable que le $\mathrm{IV}^{\mathrm{e}} \mathrm{s}$. y est beaucoup plus largement représenté. Une partie des formes présentes à Arras (bol rond Ia, vase bilobé, bol caréné VIIIa) se rapproche des productions connues dans l'officine de Labuissière (Tuffreau-Libre, 1980b). Les conditions de fouilles de ce village de potiers n'avaient pu fournir que des datations imprécises, la monnaie la plus tardive (époque constantinienne) étant de provenance douteuse. Il est désormais possible de situer la dernière période de production au moins vers le milieu du $\mathrm{IV}^{\mathrm{e}} \mathrm{s}$. Les céramiques les plus tardives de Labuissière correspondent aux formes trouvées à Arras dans le troisième quart du $\mathbf{I V}^{\mathrm{e}} \mathrm{s}$., ce qui a l'intérêt de nous donner aussi une indication sur l'écart entre la fabrication d'une céramique commune et son rejet.

\section{LES CÉRAMIQUES FINES}

Plusieurs catégories de céramiques fines existent à Arras au Bas-Empire. La céramique rouge pompéien et la terra nigra tardive sont les plus fréquentes (tabl. II). Peu courante (ici $1 \%$ maximum), la céramique micacée apparait dans le Pas-de-Calais dès le début du $\mathrm{I}^{\mathrm{er}} \mathrm{s}$. (Tuffreau-Libre, 1978). Elle ne concerne souvent que des formes rares, imitées du bronze et essentiellement retrouvées dans des contextes funéraires : Vimy (Monchy, 1977), Baraile (Tuffreau-Libre, 1989). Les quelques fragments retrouvés à Arras appartiennent à des assiettes à paroi ronde. La céramique métallescente est mieux représentée (1 à $3 \%$ ) mais reste encore très faible. Par comparaison, la sigillée atteint de 5 à $9 \%$, proportion néanmoins inférieure à celle constatée dans les niveaux du $\mathrm{I}^{\mathrm{er}} \mathrm{s}$. à Arras (Tuffreau-Libre, Jacques, 1985). La céramique peinte est représentée par quelques tessons de provenance incertaine trouvés dans des contextes de la seconde moitié du III $^{\mathrm{e}} \mathrm{s}$. (fig. 8).

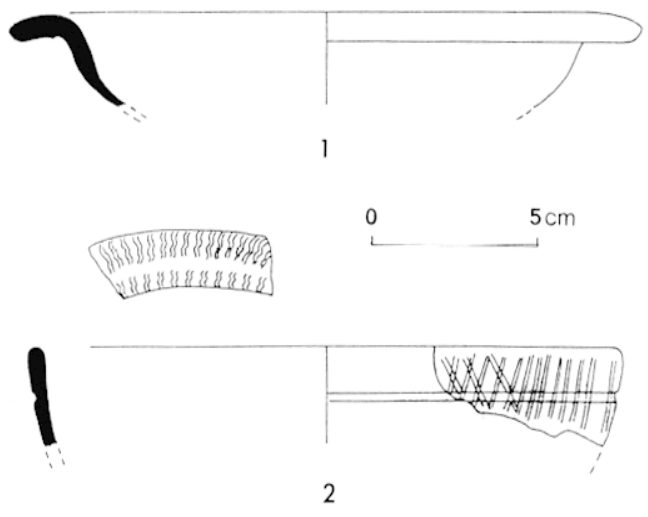

Fig. 8 - Arras, site de Baudimont : la céramique peinte.

Leur aspect rappelle les productions anglaises (Marsh, 1978), mais le petit nombre d'échantillons ne permet pas de conclusions certaines. Il faut signaler aussi la présence de tessons de céramique grise, imitant les vases en sigillée à décor incisé, dans des niveaux très différents, de la seconde moitié du III $^{\mathrm{e}}$ s., au troisième quart du Ive $^{\mathrm{e}} \mathrm{s}$.

Les céramiques fines d'importation sont donc plutôt rares, les deux catégories les plus représentées étant des productions régionales ou locales.

\section{$L a$ terra nigra tardive}

Des productions en terra nigra tardive avaient été découvertes pour la première fois en Atrébatie à Villers-au-Bois (Jacques, Tuffreau-Libre, 1978) et attribuées au III $^{\mathrm{e}} \mathrm{s}$., en l'absence d'éléments de datation précis. En fait, elles semblent apparaître à Arras vers la fin $\mathrm{du}_{\text {III }^{\mathrm{e}}} \mathrm{s}$. car elles sont signalées pour la première fois dans un puits daté de cette période. Leur représentation augmente ensuite au cours du ${ }^{2} v^{e}$ s. pour atteindre un maximum à la fin du Ive s. et décroître ensuite. Les similitudes de cette fabrication avec la terra nigra du Haut-Empire en font tout l'intérêt.

Tableau II -- Répartition des différentes catégories de céramique à Arras au IV s.

\begin{tabular}{|c|r|r|r|r|r|r|}
\hline \multirow{2}{*}{ PÉriode } & \multicolumn{2}{|c|}{ DÉBUt DU IV } \\
\hline catégorie de céramique & N.M.I. & \multicolumn{2}{|c|}{ MILIEU DU IV } \\
\hline
\end{tabular}


La terra nigra du I $^{\mathrm{er}} \mathrm{s}$. du Nord de la France se caractérise par une surface lisse et brillante, bien noire et une pâte gris clair, fine au dégraissant peu visible. Les formes de cette période sont directement copiées sur le répertoire italique pour les formes basses (assiettes), tandis que les formes hautes conservent des influences très marquées des périodes gauloises (vases ovoïdes, vases à piédestal, bouteilles). Les décors employés, peignage et guillochis, reflètent également cette double influence.

Production florissante dès la fin de la période augustéenne, elle ne se limite d'ailleurs pas à la Gaule Belgique. Elle est également présente dans le Centre, l'Ouest et semble en fait couvrir l'ensemble du territoire gallo-romain, avec des légères variantes dans les couleurs de pâtes et surtout dans les répertoires.

La terra nigra est fabriquée et diffusée durant tout le $\mathrm{I}^{\mathrm{er}} \mathrm{s}$., mais elle commence à diminuer à partir de la période claudienne. Elle persiste cependant encore dans le $\mathrm{II}^{\mathrm{e}} \mathrm{s}$., avec une restriction et une simplification des formes pour recouvrir enfin un aspect résiduel dans les niveaux du ${ }_{1 I I}{ }^{\mathrm{e}} \mathrm{s}$.

La terra nigra tardive découverte à Arras a un aspect comparable à celle du $\mathrm{I}^{\mathrm{er}} \mathrm{s}$., noir, lisse et brillant. Les décors utilisés se composent exclusivement de séries de guillochis disposés en bandes et ceux d'inspiration gauloise ont désormais disparu. Les parois des vases sont généralement plus épaisses. $\mathrm{Si}$ les analogies techniques semblent frappantes, les comparaisons s'arrêtent là car le répertoire des formes se différencie complètement de celui du Haut-Empire : il ne concerne pratiquement que des formes hautes, distinction essentielle par rapport aux productions $d u \mathrm{I}^{\mathrm{er}} \mathrm{s}$. et n'est pas original puisque la presque totalité des formes reconnues sont fabriquées indifféremment en pâte ordinaire, gris clair lustré, ou en terra nigra. Les premières formes fabriquées dérivent des vases et marmites tronconiques (fig. $6, \mathrm{n}^{\text {os }} 2,3,4,7,8$ ). Il faut y ajouter un bol à panse ronde (fig. $4, \mathrm{n}^{\circ} 10$ ). Puis, après la première moitié du Ive s., la gamme s'enrichit avec des formes bilobées, des bouteilles tronconiques, des vases à panse ronde de petite taille (fig. $7, n^{\circ s} 1,5,7,12$ ).

Une analyse des pâtes de cette production réalisée par le laboratoire du musée du Louvre a donné d'intéressants résultats que nous résumons ici, l'étude complète devant être par ailleurs publiée (Leclaire, Tuffreau-Libre, 1991). Ces analyses ont été effectuées sur un échantillonnage comprenant d'une part un lot de céramiques en lerra nigra du $\mathrm{I}^{\mathrm{er}} \mathrm{s}$. (période Auguste-Claude) provenant d'Arras et de sites campagnards voisins, d'autre part sur des céra- miques du IV $\mathbf{e}$ s. provenant du site de la Préfecture à Arras et de celui de Villers-au-Bois.

Les résultats essentiels portent sur plusieurs points. Au $\mathrm{I}^{\mathrm{er}} \mathrm{s}$., plusieurs groupes de production peuvent être distingués, qu'il s'agisse de tessons provenant de la ville ou de la campagne. Par contre, au $\mathrm{IV}^{\mathrm{e}} \mathrm{s}$., toutes les céramiques appartiennent à un groupe unique (Arras et Villers-au-Bois). D'autre part, un des groupes $d u i^{e r}$ s. a des analogies frappantes avec celui du Bas-Empire. Quelques conclusions peuvent être tirées de ces analyses qui viennent confirmer les hypothèses que nous avions au départ.

D'une part, la terra nigra tardive retrouvée à Arras peut être considérée comme la reprise, sinon la prolongation des fabrications du Bas-Empire. Il est possible que les ateliers ayant fabriqué les groupes de terra nigra identiques au $\mathrm{I}^{\mathrm{er}}$ et au $\mathrm{IV}^{\mathrm{e}} \mathrm{s}$. n'aient jamais cessé complètement d'exister. La présence dans les niveaux du milieu du $11^{\circ} \mathrm{s}$. de lessons de terra nigra, rares certes, peut être interprétée soit comme d'origine résiduelle mais aussi comme le signe d'une production très réduite mais toujours existante. Puis, à la fin du $\mathrm{III}^{\mathrm{e}}$ s., c'est une production abondante aux formes renouvelées qui réapparaît, correspondant peut-être à une reprise de l'atelier, qui peut éventuellement être replacé dans un contexte général de retour aux traditions du début du $\mathrm{r}^{\mathrm{er}} \mathrm{s}$.

La présence au Bas-Empire d'un seul groupe de production, l'abondance de cette céramique à Arras et sa diffusion dans la région voisine laissent supposer que cette fabrication est d'origine locale, peutêtre située à Arras même. En effet, la terra nigra tardive ne semble pas avoir été fabriquée à Labuissière. Les vases à panse aplatie moulurée, caractéristiques à Arras, sont absents du répertoire de cette officine, qui a certes produit des formes analogues à celles des terra nigra mais dans des pâtes ordinaires.

Il ne s'agit pas là d'un fait isolé. Des fabrications similaires existent à Saclay (Essonne) (Giganon, Tuffreau-Libre, 1981), ainsi qu'en d'autres parties de l'Ile-de-France (Jobelot, 1986). Il est possible que cette technique caractéristique $d u{ }{ }^{\text {er }} \mathrm{s}$. revive dans des formes propres à chaque région, à partir de la fin $\mathrm{du}$ III $^{\mathrm{e}} \mathrm{s}$.

\section{La céramique à enduit rouge pompéien}

Les céramiques à enduit rouge apparaissent dès la période augustéenne, où de nombreuses formes reprises $\mathrm{du}$ répertoire méditerranéen sont réalisées indifféremment en terra nigra, terra rubra et dans des pâtes beiges, partiellement peintes en rouge. A la fin du I ${ }^{\text {er }}$ s. apparaît une forme d'assiette à paroi oblique (Blicquy V) (De Laet et alii, 1972), très répandue sur tous les sites du Nord et du Pas-de-Calais. Les data- 
tions les plus tardives de cette forme ont longtemps été limitées au milieu du III $^{\mathrm{e}} \mathrm{s}$. En fait, l'abondance de cette céramique dans les niveaux de la fin du ${ }_{I I}{ }^{e}$ s. et $d u I^{e}$ s. est un fait connu à Arras, mais aussi sur d'autres sites comme celui de Graincourtles-Havrincourt (fin du IV ${ }^{e} s$.). Encore bien représentée dans la seconde moitié du IV ${ }^{e}$ s., elle ne se raréfie qu'à la fin du Ive s. Des formes dérivées, plus grossières, existent encore dans les niveaux correspondant à la phase d'abandon des casernes.

\section{LA CÉRAMIQUE GRANULEUSE}

La céramique granuleuse apparaît pour la première fois à Arras dans les couches de comblement du sanctuaire d'Attis-Cybèle (fin du troisième quart $d u I^{e}$ s.). Sa représentation reste stable à la fin du IV $v^{e}$., pour augmenter dans le premier quart du $v^{e} s$. Les formes reconnues sont peu nombreuses : assiette (Alzei 29), vase (Alzei 27) et pichet (Alzei 30) (Unverzagt, 1976) (fig. 9).

Tous les fragments ne semblent pas avoir la même provenance. Si l'Eifel semble une des origines probables, d'autres ne sont pas à exclure car des imitations locales ont pu être fabriquées, notamment en Ile-de-France, où la céramique granuleuse tardive est bien répandue (Petit, 1975). Des analyses de pâtes viendront éclaircir cette question.

\section{LA CÉRAMIQUE NON TOURNÉE}

Quelques tessons de céramique non tournée ont èté découverts sur le site d'Arras dans les niveaux du III $^{\mathrm{e}} \mathrm{s}$. Leur association avec des fragments de terra nigra, et même de terra rubra nous conduit à les considérer logiquement comme de la céramique résiduelle. Par contre, dans les niveaux correspondant au sanctuaire germanique et à d'autres structures contemporaines sont apparues des céramiques d'un type inhabituel jusqu'alors dans des contextes tardifs (fin du Ive, début du ve s.). Il s'agit de poteries façonnées à la main, dont le faciès général rappelle fortement les productions de l'Age du Fer, à la fois par les formes et les pâtes. Aucun élément ne permettait l'attribution de ces céramiques à une ancienne occupation protohistorique. A la lumière des découvertes archéologiques (sanctuaire germanique) et de l'étude des ossements humains (Blondiaux, 1990), force a été de conclure qu'il s'agissait là de fabrications attribuables à un groupe de population différent des gallo-romains, assez réduit peutêtre, compte tenu du nombre relativement limité des céramiques.
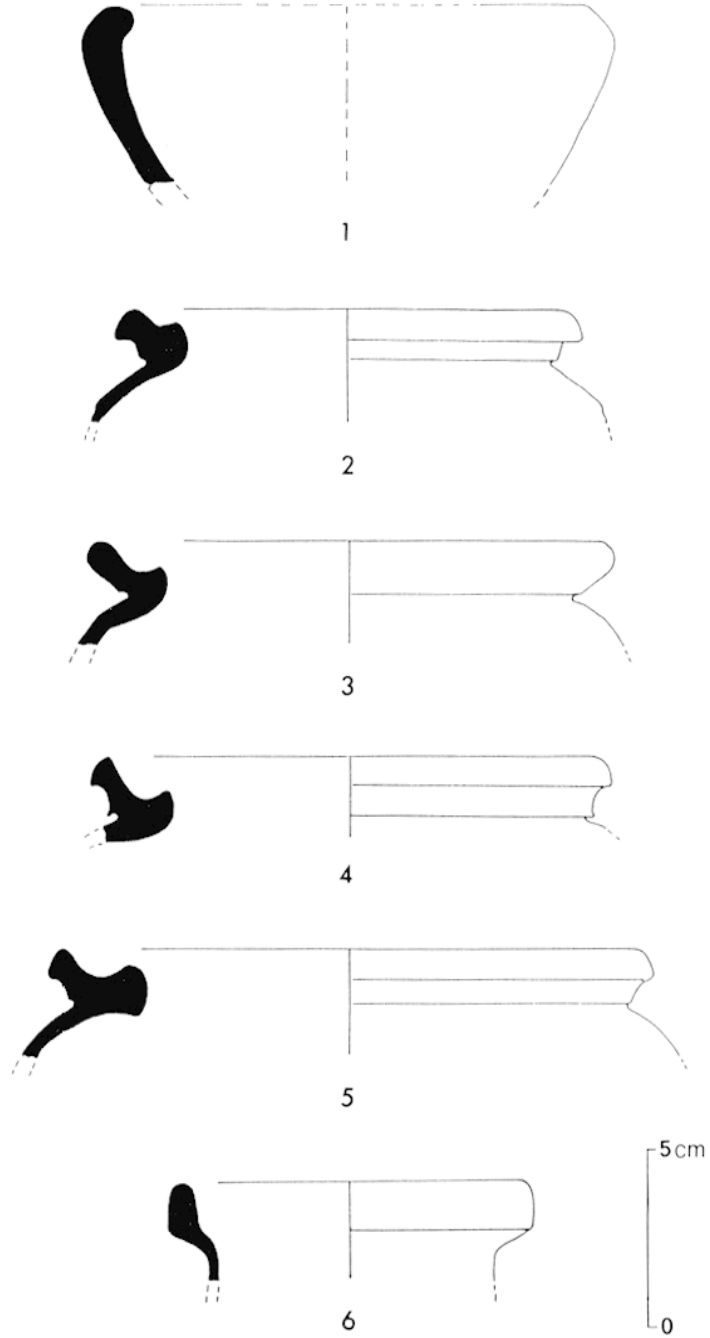

Fig. 9 - Arras, site de Baudimont : la céramique granuleuse.

Ces dernières proviennent de plusieurs structures. Certains tessons ont été reconnus dans le sanctuaire germanique et dans les couches de remblai contemporaines des casernes qui l'avaient recouvert. D'autres sont associès à la découverte des quatre squelettes de type germanique dans une fosse dépotoir de boucherie. Un autre fragment important se trouvait dans un puits. Cette répartition du mobilier dans des structures assez proches les unes des autres et de types divers suggère donc une présence étrangère sur l'ensemble du site.

Les caractéristiques d'ensemble de ces fabrications sont homogènes. La technique rappelle celle de l'époque gauloise, mais les pâtes sont mieux cuites; les argiles employées semblent d'origine régionale. La surface de certaines céramiques comme les assiettes et les vases à panse ronde est lissée et brillante, traitement que l'on retrouve sur des formes 


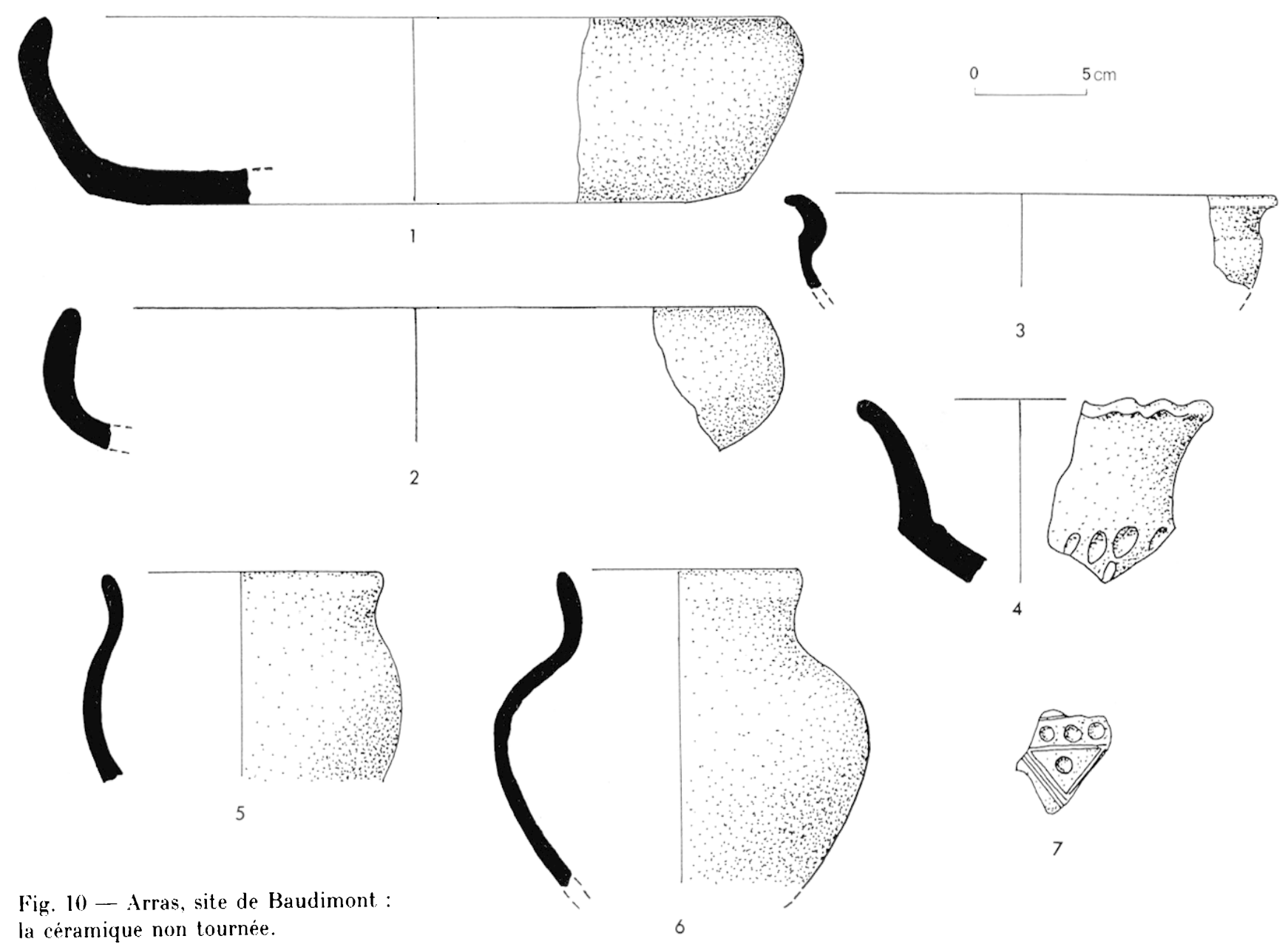

identiques dans un autre site ayant fourni de nombreuses céramiques proches, celui de Seclin (Nord). Les types sont assez réduits. Il s'agit essentiellement d'assiettes, de bols carénés et de vases à panse ronde, très proches de ceux trouvés sur les sites de Westphalie centrale, et de la Drente (fig. 10).

Les découvertes de poteries non tournées à Arras viennent s'inscrire dans un contexte plus général. En effet, des tessons comparables sont apparus récemment sur d'autres sites régionaux: Seclin, Izel-les-Hameaux, Labuissière, Boulogne-sur-Mer, tous dans des contextes de la fin du ive $\mathbf{s}$. et du début du ve s., et toujours associés à des céramiques galloromaines tardives, sans qu'aucune attribution de ces poteries non tournées à la période gauloise ne soit possible. Des ensembles identiques avaient déjà été signalés à Donk (Belgique) (Van Impe, 1983). Les nombreuses comparaisons avec les sites de Westphalie montrent que ces poteries s'apparentent fortement aux productions connues en Germanie, où les populations continuèrent à évoluer en dehors de l'influence romaine. Il est probable que de nombreuses trouvailles similaires ont été, dans la région, attribuées à l'époque gauloise en raison des caractères très proches des deux productions qui rendent leur identification difficile en dehors de tout contexte précis, ceci d'autant plus qu'il semble à Arras, comme à Seclin où à Boulogne-sur-Mer, que ces poteries aient été fabriquées à partir d'argiles régionales.

Ce lot de céramique peut donc être attribué à un groupe de population étranger à la région, venu s'installer à Arras dans le dernier quart du Iv ${ }^{e}$ s. et conservant ses coutumes et ses habitudes de vie quotidienne. La céramique ne permet pas réellement d'apprécier l'importance de ce groupe puisque nous ignorons quelle fut la proportion de céramique non tournée utilisée par rapport à l'ensemble. Néanmoins, leur pourcentage relativement peu élevé jusqu'ici suggère un nombre d'individus assez restreint.

La grande similitude entre la céramique galloromaine correspondant à cette occupation et celle qui lui est immédiatement postérieure (première phase de construction des casernes) laisse à penser que la présence de ces étrangers est de courte durée. 
Cette hypothèse est renforcée par la quasi-disparition de céramiques germaniques dans les derniers niveaux, correspondant à la destruction des casernes. Les éléments fournis par l'étude de la céramique viennent donc conforter ceux mis en évidence par la fouille et l'étude des ossements humains.

\section{LA RÉUTILISATION DE LA CÉRAMIQUE}

Les exemples de réutilisation de céramiques du Haut-Empire dans les nécropoles tardives sont nombreux. Il est plus rare de constater ce fait sur des sites d'habitats. Quelques trouvailles faites à Arras montrent que ce réemploi a été chose courante, sinon systématique, et cela en dehors du milieu funéraire.

Le cas le plus évident est celui d'un puits creusé au III $^{\mathrm{e}} \mathrm{s}$. et comblé à la fin du Ive $\mathrm{s}$. Le mobilier retrouvé dans cette structure comprend un ensemble caractéristique de la fin du $\mathrm{IV}^{\mathrm{e}} \mathrm{s}$., accompagné d'un petit lot de céramiques bien représentatif du début du ${ }^{\mathrm{e}}{ }^{\mathrm{e}}$ s., très semblable à ceux connus dans la nécropole de Baralle (fig. 11). L'association des objets (majorité de céramiques fines) suggère d'ailleurs qu'il s'agit du mobilier d'une tombe récupéré au BasEmpire, puis rejeté (après utilisation?) avec des objets cette fois contemporains de ce réemploi.
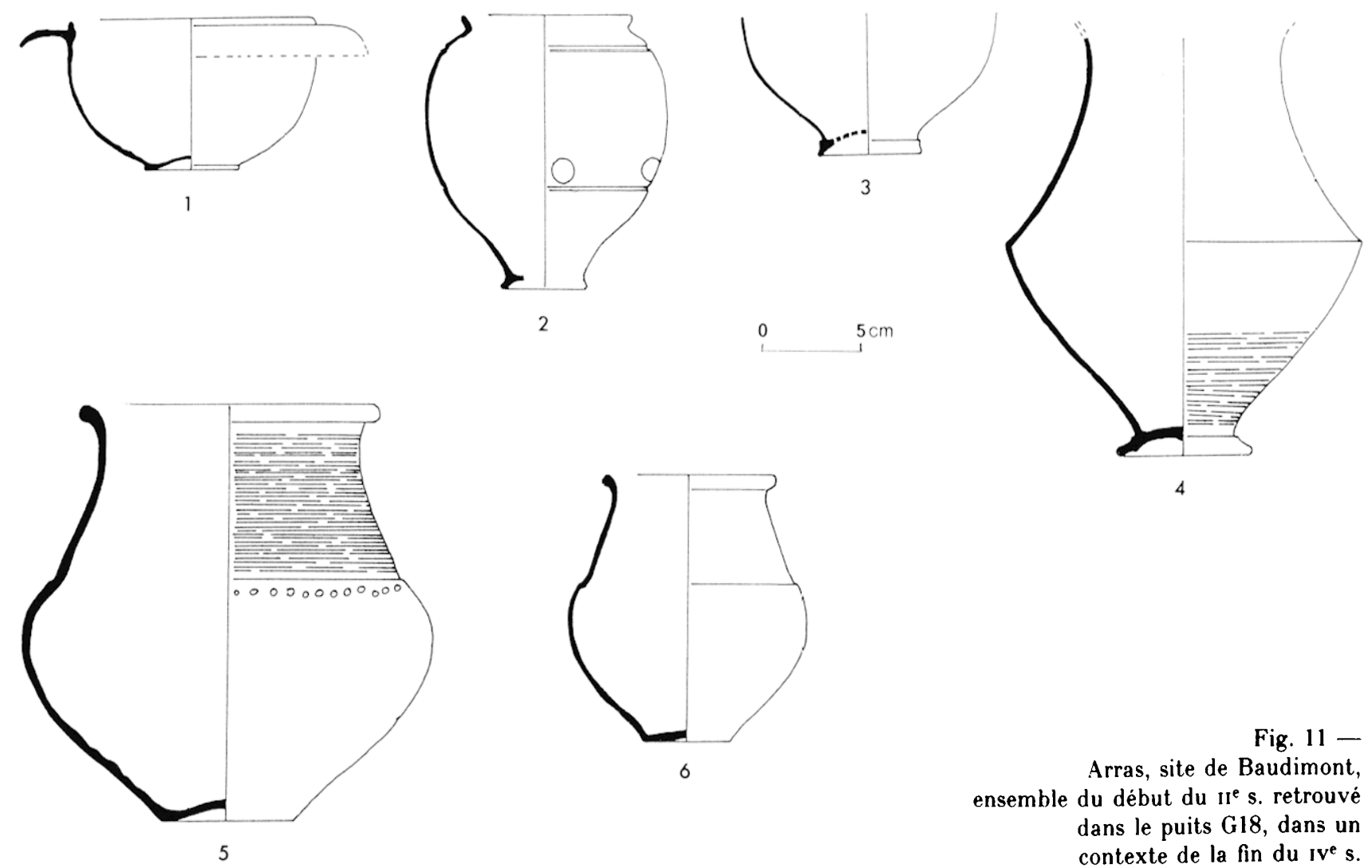

\section{ASPECTS RELIGIEUX, SOCIAUX, ÉCONOMIQUES}

\section{LA CÉRAMIQUE DU SANCTUAIRE GERMANIQUE}

La céramique trouvée dans le sanctuaire germanique a un double intérêt (fig. 12 et 13). D'une part, il s'agit d'objets correspondant cette fois à une phase d'occupation qui peut être datée de façon précise, puisqu'elle se situe entre deux niveaux de comblement, celui du sanctuaire d'Attis et Cybèle (dans les années 370 ) et celui correspondant au dépotoir de la première phase des casernes (vers les années 380 ).

Fig. 11 Arras, site de Baudimont, ensemble du début $d u{ }^{2}{ }^{e} s$. retrouvé dans le puits $\mathrm{G} 18$, dans un contexte de la fin du ive $s$. 

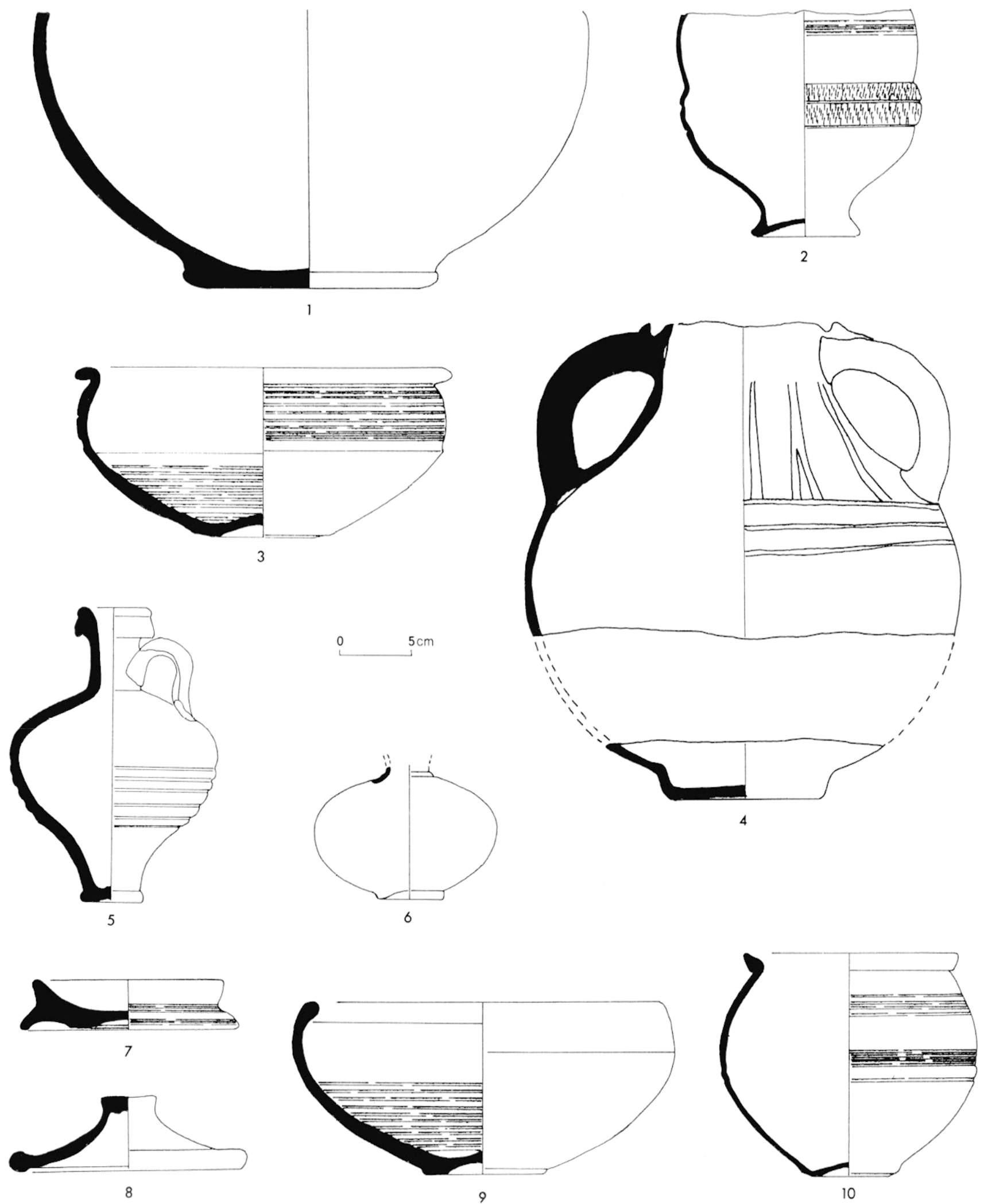

Fig. 12 - Arras, site de Baudimont : la céramique du sanctuaire germanique. 

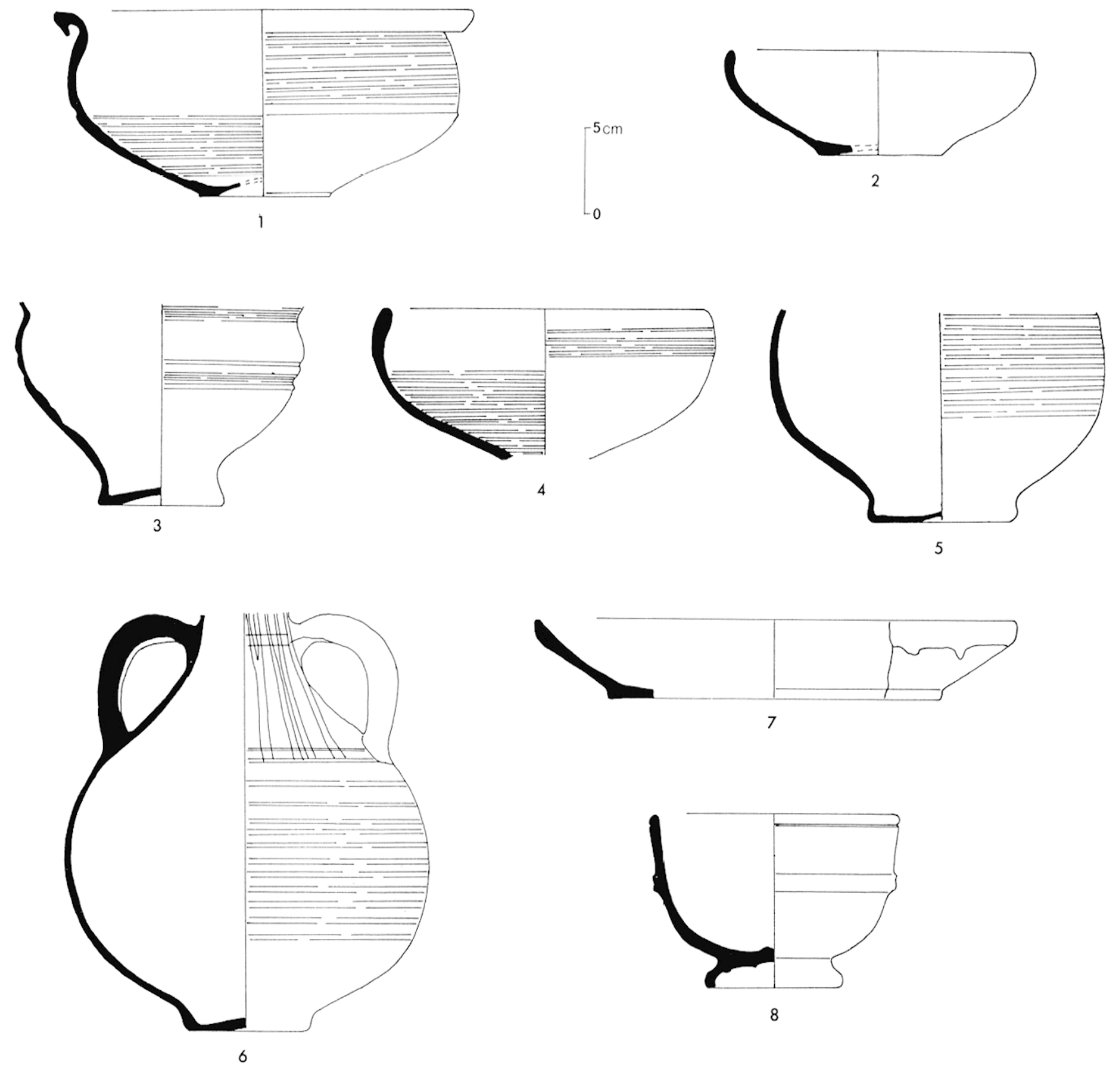

Fig. 13 - Arras, site de Baudimont : la céramique du sanctuaire germanique.

De plus, si ces objets sont l'instantané partiel mais précis d'une courte période, ils se rattachent à des structures particulières puisque nous sommes ici dans un milieu funéraire et religieux bien individualisé. Cette céramique est donc à la fois le reflet des répertoires du troisième quart $d u$ IV $^{\mathrm{e}} \mathrm{s}$. et celui des coutumes d'un groupe de population que tout semble désigner comme d'origine étrangère.

La céramique du sanctuaire germanique ne présente pas d'originalité flagrante par rapport à celle des autres structures. Le répertoire des formes en céramique commune est semblable à celui correspon- dant au comblement du sanctuaire d'Attis-Cybèle ainsi qu'à celui de la première phase des casernes, ce qui suggère une phase d'occupation très courte. Le mobilier se compose de bols à panse ronde, bols carénés, vases bilobés et à panse aplatie, de cruches essentiellement, formes courantes sur le site de Baudimont durant toute la seconde moitié du IV $\mathbf{s}$.

Le choix des objets n'est cependant pas laissé au hasard. Ainsi, les céramiques de l'aire d'offrande sont toutes de couleur rouge et appartiennent à des catégories de céramiques fines : sigillée, rouge pompéien, imitation de sigillée. Ln type de céramique, 
par ailleurs plutôt rare sur l'ensemble du site est bien représenté. Il s'agit de cruches en terre rouge, avec un décor de bandes lustrées sur l'épaule et sur la panse. ici toutes dépourvues de col. De même, on note la présence d'un bol à collerette, forme également peu représentée à Arras. La préférence va d'autre part aux bols et aux cruches, objets privilégiés pour contenir nourriture et boisson. Le type de matériel choisi s'apparente donc au mobilier funéraire déposé dans les tombes (Van Doorselaer, 1967). Il faut donc souligner le fait que si les rites observés dans le sanctuaire germanique nous renvoient vers les périodes celtiques et les peuplades de Germanie, le choix des objets utilisés pour les accomplir s'est fait en conformité avec les usages des Gallo-romains du Ives.

Le traitement des objets et leur disposition rappcllent également les rites pratiqués dans les tombes d'époque gallo-romaine. I es céramiques des fosses se retrouvent en fragments ou complètes, mais, dans ce cas, elles sont rendues inutilisables par la présence de fêlures ou de trous. Le bol imitant la sigillée montre une collerette brisée, dont les bords restants sont soigneusement égalisés. Le bris intentionnel de la céramique, d'origine préromaine, est attesté dans de nombreuses nécropoles gallo-romaines. Il est connu aussi dans le Nord de la France, à Noyelles-Godault (Demolon, Tuffreau-Libre, 1976), où un puits contenait deux séries de squelettes séparés par un niveau stérile, et en dessous, une couche de tessons dont les groupements indiquent un bris préalable à l'enfouissement. Ces coutumes, symbolisant la fin de la vie terrestre, sont attestées dans des points très divers du territoire gallo-romain.

La disposition à l'envers des céramiques de l'aire d'offrande, identique à celle des crânes humains et animaux, suggère aussi un rite bien précis, attesté dès l'Age du Bronze et présent dans certaines tombes gallo-romaines, où les urnes recouvrent les ossements comme un couvercle. Un puits funéraire du milieu du $\mathrm{r}^{\mathrm{er}} \mathrm{s}$. à Cazères (HauteGaronne) (Manière, 1966) montre des dispositions analogues : de nombreux fonds de poterie retournés abritent des petits tas d'ossements d'animaux. Cette disposition suggère l'existence d'une vie souterraine du défunt, rejoignant les divinités chthoniennes.

La céramique témoigne donc de coutumes funéraires et religieuses présentes surtout au début de la période gallo-romaine, remises ici en évidence par un groupe de population probablement germanique, ce qui confirmerait l'origine préromaine de la plupart de ces coutumes.

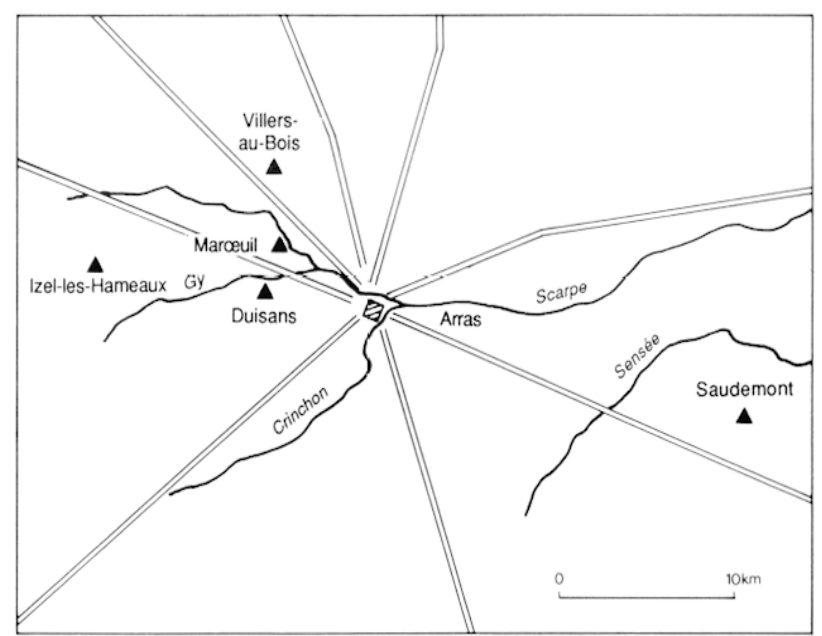

Fig. 14 - Les sites campagnards de la région d'Arras.

\section{VILLE, NÉCROPOLES ET CAMPAGNES}

Il nous a semblé intéressant d'établir des comparaisons entre les ensembles d'Arras et ceux trouvés dans des sites de la campagne environnante (fig. 14), ainsi qu'avec des objets d'époque similaire mais provenant de nécropoles.

La céramique trouvée dans les habitats campagnards de la région d'Arras montre une évolution semblable à celle de la ville. Ces mobiliers, issus de structures diverses (fosses, fossés de villa), sont beaucoup moins représentatifs que ceux d'Arras en raison de leur faible importance quantitative. De ce fait, ils n'autorisent que des comparaisons limitées mais n'en donnent pas moins des indications réelles, reflétant les tendances générales.

L'ensemble de Villers-au-Bois peut être considéré comme le plus ancien en raison d'un pourcentage important de terra nigra tardive (vases et marmites tronconiques) (fig. 15 et tabl. III). La présence de bol rond Ia et de vase rond XIa, en faible quantité, indique une datation proche de celle de la Préfecture (milieu du Ive s). Le site de Marœuil apparaît un peu plus tardif (disparition des terra nigra, représentation importante des bols ronds Ia et des vases ronds XIa). L'absence de vase bilobé ne permet pas une datation trop avancée dans le $\mathrm{Iv}^{\mathrm{e}} \mathrm{s}$. Par contre, la présence importante des variantes les plus tardives de ce dernier type sur les sites de Saudemont et de Duisans, toujours associées aux bols ronds Ia et aux vases à panse ronde $\mathrm{XIa}$, daterait ces deux sites dans le premier tiers du ves.

Quelques différences entre la ville et les campagnes sont sensibles à plusieurs niveaux. 


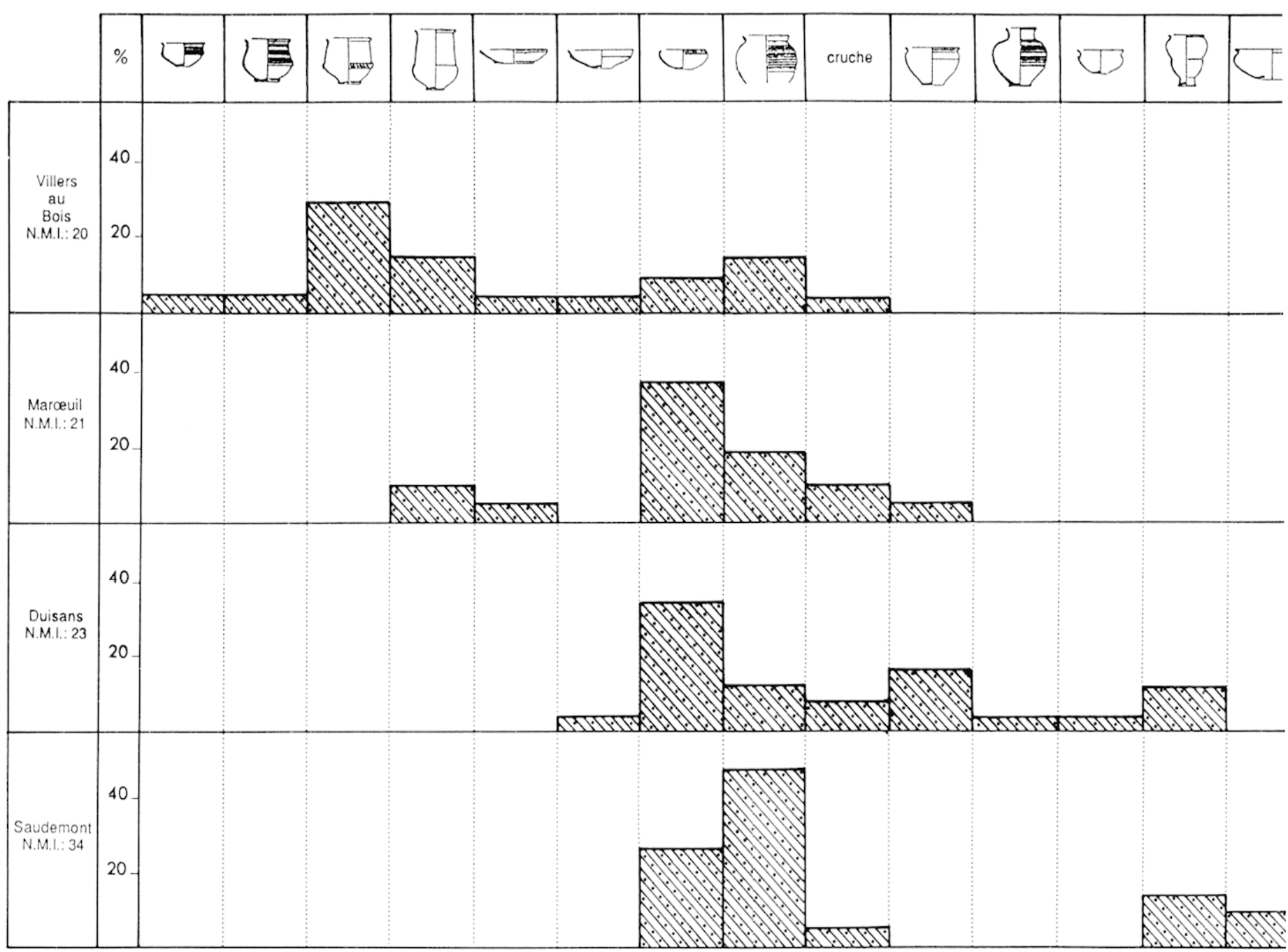

Fig. 15 - Répartition de la céramique sur les sites ruraux de la région d'Arras.

Tableau III - Proportions des différents types de céramiques connus sur les sites ruraux (en pourcentages).

\begin{tabular}{|c|c|c|c|c|}
\hline SITE & Villers-AU-Bois & MAR(EUIL & Duisans & SAUDEMONT \\
\hline ass. POIIIa $\ldots \ldots \ldots \ldots \ldots$ & 5 & 5 & - & - \\
\hline bol rond $\ldots \ldots \ldots \ldots \ldots \ldots$ & 5 & 14 & - & 3 \\
\hline bol RIa .............. & 10 & 38 & 35 & 26 \\
\hline bol RVIb $\ldots \ldots \ldots \ldots \ldots \ldots$ & - & - & - & 9 \\
\hline bol CIIa ............... & 5 & - & - & - \\
\hline bol CVIIIa $\ldots \ldots \ldots \ldots \ldots$ & 5 & - & 4 & - \\
\hline bol CiXIIa .............. & 4 & - & - & - \\
\hline marmite PAIIa ........... & 5 & - & - & - \\
\hline marmite PA $\ldots \ldots \ldots \ldots \ldots$ & 30 & - & - & - \\
\hline marmite CXVIa $\ldots \ldots \ldots \ldots$ & - & 5 & 17 & - \\
\hline vase tronc. .............. & 15 & 10 & - & - \\
\hline vase $\mathrm{RId} \ldots \ldots \ldots \ldots \ldots$ & - & - & 4 & - \\
\hline vase $\mathrm{PRXIa} \ldots \ldots \ldots \ldots$ & 15 & 19 & 13 & 47 \\
\hline vase bilobé $\ldots \ldots \ldots \ldots \ldots$ & - & - & 13 & 12 \\
\hline cruche $\ldots \ldots \ldots \ldots \ldots \ldots$ & 5 & 10 & 9 & 3 \\
\hline
\end{tabular}


Tout d'abord, les types de céramiques présents en campagne sont beaucoup moins variés. Les formes se limitent à celles les plus représentatives de la période : marmites en terra nigra pour Villers-auBois, bols ronds Ia, vases ronds XIa, vases bilobés, pour les autres sites. Quelques céramiques différentes apparaissent : cruche, bol caréné, vase tronconique, assiette rouge pompéien, en fonction de la période. Même si l'on tient compte de la faiblesse de l'échantillonnage par rapport à celui de la ville, il n'en demeure pas moins que la céramique semble moins diversifiée et se rattache surtout à des répertoires régionaux.

Les comparaisons des différentes catégories de céramiques viennent confirmer ces premières données. Les proportions de céramiques fines sont en moyenne plus importantes à Arras, de même que certaines catégories faiblement représentées en ville (céramique métallescente, céramique micacée) sont absentes (tabl. IV).

Ces décalages s'expliquent aisément par le rôle de carrefour commercial joué par la ville, où parvenaient non seulement les productions strictement régionales, mais aussi les importations des régions voisines et plus éloignées, alors que les campagnes avaient surtout un approvisionnement local. D'autre part, les céramiques fines se commercialisaient plus aisément en ville, où la demande était plus importante. Par contre, la céramique sigillée montre une proportion identique en ville et en campagne, de 5 à 9. Elle est aussi, globalement, la céramique fine la plus représentée en campagne. On peut supposer qu'à cette époque tardive, elle demeure la céramique de luxe par excellence, celle peut-être la plus longtemps conservée.

Des comparaisons avec le $\mathrm{I}^{\mathrm{er}} \mathrm{s}$. montrent que la céramique fine a toujours été plus faiblement représentée dans les campagnes. Sa présence à Arras diminue au $I^{e} s$. mais est encore importante. Globalement, la production et le commerce de la céramique semblent encore actifs dans la ville à la fin du BasEmpire, mais peut-être dans un cadre régional plus limité.

Si les contrastes entre ville et campagne, bien qu'encore difficiles à apprécier en raison de la faiblesse de ces données, sont finalement de peu d'importance, et s'inscrivent dans une logique économique et sociale, il n'en est pas de même pour le mobilier connu dans les nécropoles contemporaines des niveaux d'habitat d'Arras qui montrent au contraire des décalages importants.

Certains d'entre eux s'expliquent aisément. Ainsi, la céramique sigillée est, dans le cas des sépultures, bien représentée, ainsi que les autres céramiques fines en général $(27 \%$ et $12 \%$ à Noyellessur-Mer (Piton, Marchand, 1978). Il s'agit là d'un fait constaté déjà au $\mathrm{I}^{\mathrm{er}} \mathrm{s}$. de façon générale, traduisant la volonté de déposer dans les tombes un mobilier de qualité. De même, des différences typologiques sont simplement dues aux usages funéraires, privilégiant certains types d'objets plutôt que d'autres, afin de présenter les mets et boissons accompagnant le défunt. C'est ainsi que les cruches et les bouteilles, peu représentées dans les couches d'habitat, atteignent une proportion importante dans les nécropoles. La céramique de ces dernières apparaît aussi moins diversifiée que celle des habitats, renvoyant ainsi une image quelque peu faussée de la réalité. Le mobilier retrouvé à Arras dans le sanctuaire germanique illustre bien ce cas.

Par contre, certaines contradictions entre les datations proposées par les nécropoles d'une part, les habitats de l'autre, laissent entrevoir quelques problèmes chronologiques. Le mobilier de la nécropole d'Oudenburg (Mertens, Van Impe, 1971) montre de nombreuses analogies avec celui d'Arras. Les datations proposées essentiellement en fonction de la numismatique s'arrêtent au plus tard au débuit du $v^{e}$ s., la majeure partie des sépultures étant placée dans la seconde moitié du $I^{e} \mathrm{~s}$. Or le répertoire des

Tableau IV - Répartition des différentes catégories de céramique sur les sites campagnards.

\begin{tabular}{|c|c|c|c|c|c|c|c|c|}
\hline Site & \multicolumn{2}{|c|}{ MAREUIL } & \multicolumn{2}{|c|}{ SAUDEMONT } & \multicolumn{2}{|c|}{ Villers-AU-Bois } & \multicolumn{2}{|c|}{ Duisans } \\
\hline catégorie de céramique & N.M.I & $\%$ & N.M.I & $\%$ & N.M.I & $\%$ & N.M.I & $\%$ \\
\hline sigillée & 3 & 8 & 3 & 5 & 2 & 6 & 4 & 9 \\
\hline céramique à enduit rouge .... & 1 & 3 & 0 & 0 & 1 & 3 & 0 & 0 \\
\hline terra nigra $\ldots \ldots \ldots \ldots \ldots$ & 0 & 0 & 0 & 0 & 4 & 11 & 0 & 0 \\
\hline céramique ordinaire $\ldots \ldots \ldots$ & 35 & 89 & 53 & 95 & 29 & 80 & 39 & 91 \\
\hline TOTAL. & 39 & 100 & 56 & 100 & 36 & 100 & 43 & 100 \\
\hline
\end{tabular}


céramiques correspond essentiellement à celui trouvé dans les niveaux les plus récents à Arras et datés dans le premier quart du $\mathrm{v}^{\mathrm{e}} \mathrm{s}$. II faut de plus signaler que certains types présents à Oudenburg sont encore inconnus à cette époque à Arras. On pourrait objecter la distance entre les deux sites, mais un examen du mobilier des tombes tardives de la nécropole de Dourges (Pas-de-Calais), très proche d'Arras, montre une conformité frappante avec celui d'Oudenburg. Ces faits laissent supposer une représentation importante dans les nécropoles de céramiques résiduelles des périodes précédentes, de mème qu'ils suggèrent une distorsion entre les datations données par les monnaies et la céramique. Le mobilier de la nécropole de Noyelles-sur-Mer, datée essentiellement du troisième quart du $\mathrm{IV}^{\mathrm{e}} \mathrm{s}$., présente le même décalage avec celui provenant de structures d'habitat théoriquement contemporaines (Bayard, Fournier, 1978). Cette constatation avait déjà été faite pour le $\mathrm{r}^{\mathrm{er}} \mathrm{s}$. (Tuffreau-Libre, 1989), mais le manque de mobilier provenant de fouilles d'habitats tardifs ne nous avait pas permis jusqu'à maintenant d'apprécier le caractère disparate des séries déposées dans les tombes du Bas-Empire, sauf dans le cas de réemplois manifestes, cela nécessitant une connaissance précise des répertoires régionaux et de leur datation.

Les objets usés, réparés avec des agrafes en fer, déposés à coté d'autres manifestement plus récents, sont choses courantes dans les sépultures du $\mathrm{I}^{\mathrm{er}} \mathrm{s}$. (Vaulx-Vraucourt, Baralle, Vimy). Le fait se constate aussi au IV's. (Caron, Monchy, 1990). Les milieux funéraires peuvent donc présenter des faciès céramiques très antérieurs à leur date véritable. En fait, la coexistence, presque systématique et souvent difficile à déceler dans les tombes d'objets anciens et d'autres contemporains de leur enfouissement, empêche des conclusions systématiques puisque de nombreux cas de figure sont possibles. Il est aussi intéressant de constater que si la proportion de céramique sigillée est proche au $\mathrm{I}^{\mathrm{er}} \mathrm{s}$. et au $\mathrm{IV}^{\mathrm{e}} \mathrm{s}$. dans les habitats, elle est par contre très différente dans les nécropoles entre les deux époques: aucune céramique sigillée dans les sépultures avant la fin du $\mathrm{r}^{\mathrm{er}} \mathrm{s}$., alors que son taux de représentation est d'environ $30 \%$ à la fin du IV $^{\mathrm{e}} \mathrm{s}$. Le dépôt dans les sépultures n'est donc pas lié à une présence plus ou moins abondante de sigillée à une période donnée. S'agit-il d'une évolution des usages, la céramique sigillée s'intégrant peu à peu aux coutumes des Gallo-romains du Nord, ou faut-il y voir un décalage dù à la très longue conservation des céramiques sigillées avant leur dépòt dans les tombes? S'il en est ainsi, le schéma du $\mathrm{I}^{\mathrm{er}} \mathrm{s}$. pourrait s'appliquer aussi au $\mathrm{IV}^{\mathrm{e}} \mathrm{s}$. Dans ce dernier cas, la céramique sigillée en milieu funéraire poserait des problèmes similaires à celui des monnaies, puisque des différences de cinquante ans à un siècle pourraient être couramment envisagées. La céramique commune apparaîtrait comme la catégorie d'objets la moins longtemps conservée avant son dépôt dans les tombes.

Ces comparaisons des datations, fournies par les habitats d'une part et les nécropoles de l'autre, montrent que ces dernières apparaissent de moins en moins comme des ensembles de référence pour les études chronologiques. Il serait prudent de privilégier leur étude dans le cadre des aspects religieux et sociaux. En dernier lieu, il est bien évident qu'il devient plus qu'hasardeux de dater les habitats avec des chronologies funéraires.

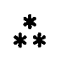

Cette étude préliminaire de la céramique tardive à Arras nous permet donc d'ajouter de nouveaux éléments à l'étude de la céramique régionale et d'aborder quelques problèmes à la fois méthodologiques et généraux.

L'examen typologique et quantitatif des céramiques apporte des précisions chronologiques de plus en plus fines puisque nous pouvons suivre l'évolution des répertoires au quart de siècle près, et mème parfois de décennie en décennie. Il devrait être possible à Arras, et dans sa région, de dater de façon plus précise les ensembles du Bas-Empire, ce qui nous conduira à progresser sensiblement dans la connaissance de cette période encore mal connue car toutes les conclusions historiques s'articulent autour de la chronologie.

En effet, ces données modifient quelque peu la vision générale que nous avions de la fin du $\mathbf{~ v}^{e} \mathrm{~s}$. puisqu'il semble que cette époque soit bien représentée sur de nombreux sites et villes romaines dont les chronologies sont simplement inexactes. De nombreuses formes voient leurs datations poussées très loin dans le Bas-Empire et la continuité entre le IV ${ }^{e}$ et le $v^{\prime \prime}$ s., bien mise en évidence ici, nous permet d'entrevoir le passage progressif du monde galloromain à l'époque mérovingienne. Encore une fois, les difficultés que nous avons à établir une chronologie de la céramique du $\mathrm{v}^{e} \mathrm{~s}$. tiennent peut-être plus à des décalages dus à des datations presque exclusivement établies d'après les milieux funéraires qu'à des absences réelles de matériaux. Les habitats nous donnent une image à la fois plus juste et plus variée des répertoires.

Du point de vue économique, la céramique ne témoigne pas d'un changement rapide, ni d'une 
récession brutale à la fin du Bas-Empire car elle n'y apparait pas de mauvaise qualité, ni spécialement appauvrie. On assiste mème à la renaissance de fabrications fines bien connues au Haut-Empire (terra nigra tardive). Les courants commerciaux ont une importance égale à celle du I ${ }^{\text {er }}$ s., avec une prépondérance très marquée des produits régionaux. II est probable qu'il faille attendre la fin du premier tiers $d u v^{r} s$. pour enregistrer des transformations notables.

Tous ces éléments viennent confirmer l'importance fondamentale de la céramique car la connaissance des répertoires régionaux apparaît de plus en plus comme une condition indispensable à la progression d'études plus générales. Il faut pour cela souligner la nécessité de disposer pour les datations des céramiques de sites de référence fiables. Dans ce domaine, il devient d'autre part essentiel de prendre en compte, en plus des caractères purement typologiques, divers éléments: comptage par forme, appréciation des durées d'utilisation, aspects résiduels, types de structure envisagés. Enfin, il apparaît prudent de limiter les implications chrono-logiques à des régions restreintes. En effet, les schémas que nous avons présentés ici sont valables pour un site et une région donnée. Ils peuvent être identiques ou différer sensiblement ailleurs.

\author{
Marie Tuffreau-Libre \\ et Alain J ACQUES
}

\section{ANNEXE \\ DESCRIPTION DETAILLEE DES FIGURES}

Cette partie comprend une description précise des céramiques présentées sur les figures 4 à 13 , que nous n'avons pas voulu intercaler dans l'étude de synthèse pour en rendre la lecture plus aisée.

C'est aussi dans un but de simplification que nous avons désigné les différents niveaux datés par des phases :

- phase I (niveau de construction du temple d'Attis-Cybèle, seconde moitié du $\mathrm{III}^{\mathrm{e}} \mathrm{s}$.);

- phase II (Préfecture, milieu du Iv $\mathrm{s}$.);

- phase III (niveau de destruction du temple d'Attis-Cybèle, vers 375);

- phase IV (première occupation des casernes, $380-390)$;

- phase V (deuxième occupation des casernes, de 390 au premier quart du $v^{e} \mathrm{~s}$.).

La description des types est donc suivie de l'indication de leur appartenance à une ou plusieurs de ces différentes phases ou à une structure plus précise.

\section{LES FORMES DE CÉRAMIQUE CONNUES Aे ARRAS (FIG. 4)}

No 1 : assiette à paroi ronde, lèvre s'affinant à l'extrémitè, fond légèrement concave; couleur externe grise, intérieur jaune, doré au mica (Munsell Soil Color Charts 10 YR 6;4); pâte à dégraissant sableux, bien cuite.

Contexte : puits G18 (troisième quart du Ives.).

Nos 2 à 4 : assiettes à enduit rouge; parois obliques, lèvres allongées, s'affinant à l'extrémité; pieds droits, arrondis à la base ; fonds plats; couleur rouge à l'intérieur et sur la lèvre (10R 4/8); pâte beige à dégraissant sableux, bien cuite.

Type II Ia des assiettes à panse oblique, phases I à V.

No 5 : assiette carénée de type Ila; lèvre rentrante; partie supérieure oblique; partie inférieure carénée; petit pied; fond concave; couleur gris clair $(2,5 \mathrm{YR}$ N3/); pâte grise, à dégraissant sableux, bien cuite. L'ensemble est décoré de séries de bandes lissées parallèles.

Phase I.

No 6 : assiette carénée de type IIa, lèvre rentrante, arrondie ; paroi carénée; fond concave ; couleur gris foncé $(2,5 Y \mathrm{R} N 3 /)$; pâte grise à dégraissant sableux, bien cuite.

Phases II à V. 
No 7 : bol rond de type XIIIa ; petite lèvre arrondie ; panse ronde; pied rond; fond légèrement creusé; couleur noire $(2,5 \mathrm{YR} \mathrm{N} 3 /)$; pâte grise, à dégraissant sableux, bien cuite.

Phases II, III, puits G 18, troisième quart du IV"s.

No 8 : bol rond de type IVb; lèvre arrondie, rentrante; panse ronde; petit pied arrondi; fond concave; couleur noire (2,5YR N3/); pâte grise à dégraissant sableux, bien cuite. La lèvre et l'intérieur du bol sont décorés de bandes lissées parallèles.

Phases I, II.

No 9 : bol rond de type Ia; lèvre arrondie; panse ronde; fond concave; couleur grise (7,5YR N6/) pâte grise, à dégraissant sableux, bien cuite. De nombreux exemplaires sont décorés d'une série de bandes lissées en haut de la lèvre.

Phases II, III, IV, V, sanctuaire germanique.

No 10 : bol rond de type Va en terra nigra; lèvre allongée, retombant sur la panse ronde; petit pied arrondi; fond creusé, marqué d'un anneau saillant; couleur noire $(2,5 \mathrm{YR} \mathrm{N} 3 /)$; pâte grise à dégraissant sableux, bien cuite.

Type connu en pâte ordinaire et en terra nigra ; phases II, III, IV.

No 11 : variante du type précédent; lèvre recourbée; panse ronde; petit pied arrondi; fond concave; couleur grise (10YR 5/1); pâte grise à dégraissant sableux, bien cuite.

Phase II.

No 12 : bol à lèvre arrondie, épaisse, panse ronde; pied haut, oblique, se terminant en arrondi; fond creusé; couleur grise (10YR 4/1); pâte grise, à dégraissant sableux, bien cuite.

Dérivé du type 320 de Chenet, phase III.

No 13 : bol à lèvre épaisse, en crochet replié; col oblique, bombé, se terminant par une moulure; panse carénée; petit pied arrondi; fond concave; couleur grise $(7,5 Y R \quad N 4 /)$; pâte grise, à dégraissant sableux, bien cuite. Le col est décoré d'une bande lissée.

Phase II.

\section{LES FORMES DE CÉRAMIQUE CONNUES A ARRAS (FIG. 5)}

No 1: bol caréné de type XVIc; lèvre repliée en crochet; panse carénée; petit pied arrondi; fond concave; couleur grise $(2,5 \mathrm{Y} \mathrm{N} 5 /, \mathrm{N} 4 /)$; pâte grise, à dégraissant sableux, bien cuite. Le haut de la panse est décoré de bandes lissées parallèles.

Phases I, II, III, IV.
No 2 : bol caréné; lèvre en crochet; col droit, légèrement bombé; couleur gris-bleu $(7,5 \mathrm{YR}$ N5/); pâte grise, à dégraissant sableux, bien cuite. Le col est orné de bandes lissées entrecroisées.

Phase I.

$N^{\wedge s} 3$ à 6 : bols carénés de type XIc; lèvres repliées en crochet; cols bombés, plus ou moins moulurés; panses carénées; pieds légèrement arrondis ou obliques; fonds concaves; couleur gris à gris foncé;

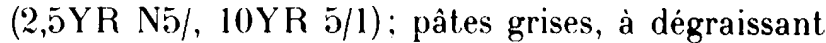
sableux, bien cuites. Le col est généralement décoré de bandes lissées parallèles, ainsi que parfois l'intérieur du bol.

Phases II, III, IV, V.

No 7: bol caréné de type VIla; lèvre allongée, recourbée en crochet; col oblique; panse carénée; petit pied arrondi; fond concave; couleur gris-bleu; pâte noirc; à dégraissant sableux, bien cuite.

Phase I.

Nos 8 et 9 : bols carénés de type VIIIa; lèvres arrondies, prolongées par un col concave; panses carénées; petits pieds arrondis; fonds concaves; couleur grise $(7, \overline{5} \mathrm{YR} \mathrm{N} 4 /, \mathrm{N} 3 /)$; pâte grise à dégraissant sableux, bien cuite. Le col et l'intérieur du bol sont parfois décorés de bandes lissées parallèles.

Phases IV et V.

No 10 : bol à collerette; lèvre épaisse, arrondie; collerette à mi-hauteur de la panse; petit pied arrondi ; fond creusé; couleur gris foncé (7,oYR N3/); pâte grise à dégraissant sableux, bien cuite.

Phases III à V.

Nos 11 et $12:$ marmites à panse aplatie de type IIa; lèvres repliées en crochet; panses aplaties, moulurées; pieds obliques; fonds creusés; couleur grise $(7,5 \mathrm{YR}$ $\mathrm{N} 5 /, \mathrm{N} 4 /)$; pàte grise, à dégraissant sableux, bien cuite. La panse est ornée de séries de bandes lissées parallèles ou recoupées de bandes lissées entrecroisées.

Phases I, II, III.

\section{LES FORMES DE CÉRAMIQUES CONNUES A ARRAS (FIG. 6)}

No 1 : vase à panse aplatie de type $\mathrm{Ib}$; lèvre repliée en crochet; panse aplatie, moulurée; petit pied arrondi; fond concave; couleur gris clair $(2,5 \mathrm{YR}$ $\mathrm{N}$ (5/); pâte grise, à dégraissant sableux, bien cuite. La partie supérieure est ornée de bandes lissées parallèles.

Phases I, II, III.

No 2 : vase à panse aplatie de type Ia en terra nigra, lèvre fine, repliée en crochet, suivie d'une moulure 
très mince; panse aplatie, moulurée; petit pied arrondi : fond concave, portant un anneau protubérant: couleur noire $(7, \overline{\mathrm{o}} \mathrm{YR} \times 2, \overline{\mathrm{y}} /)$ : pâte noire, à dégraissant sableux, bien cuite.

Phase IV.

Nos 3 et 4 : marmites ; lèvres en crochet; panses aplaties portant au centre une large moulure; pieds arrondis; fonds creusés, portant un anneau en relief ; couleur noire (terra nigra, 2,5Y N5̄i) et marron (10YR 6/2); pâte à dégraissant sableux, bien cuite. La moulure centrale est généralement décorée de séries de guillochis réguliers.

Phases II, III, IV.

No 5 : marmite; lèvre repliée en crochet; panse aplatie, moulurée ; pied mouluré ; fond concave ; couleur grise (2,5YR N5/); pâte grise, à dégraissant sableux, bien cuite. La partie supérieure est décorée de bandes lissées parallèles. Celle forme est un intermédiaire entre les marmites (nos 3 et 4 ) et les vases tronconiques (nos 9 et 10 ). Elle est réalisée en terra nigra tardive.

Phases II, III, IV.

No 6 : vase à lèvre en crochet, suivie d'une moulure fine; panse aplatie; petit pied arrondi; fond concave; couleur grise (10YR $5 / 1)$; pàte grise, à dégraissant sableux, bien cuite.

Phase III.

Nos 7 et 8 : vases tronconiques de type IId; lèvres en crochet; cols tronconiques; panses rondes moulurées; petits pieds arrondis ; fonds concaves; couleur grise (10YR 5/1, 2,5YR N3/); pâte grise, à dégraissant sableux, bien cuite. La moulure de l'exemplaire n" 8 est décorée de guillochis.

Phases II, III, IV.

No 9 : vase tronconique; lèvre recourbée en crochet; col bombé ; panse carénée; pied oblique, arrondi au sommet; fond concave; couleur gris-bleu (7,5YR $\times 5 /)$; pâte grise, à dégraissant sableux, bien cuite. Le col est orné de bandes lissées parallèles. Il s'agit de la variante la plus tardive des vases tronconiques.

Phases II, III, IV.

No 10 : vase en terra nigra tardive; lèvre épaisse, en crochet; le passage du col à la panse n'est plus marqué que par une rainure; pied arrondi; fond creusé ; couleur noire (2,5Y $\mathrm{Y} 3 /$ ) ; pâte grise, à dégraissant sableux, bien cuite. Le col est orné de bandes lissées parallèles.

Phase IV.

Nos 11 et 12 : vases à panse ronde de type XIa; lèvres en crochet; panses rondes, moulurées au centre; couleur grise (2,jYR N4/, 10YR う/1); pâte grise, à dégraissant sableux. La panse est décorée de trois séries de bandes lissées. Phases II, III, IV, V.

\section{LES FORMES DE CÉRAMIQUES CONNUES À ARRAS (FIG. 7)}

No 1 : vase bilobé de type IIc en terra nigra, lèvre fine, arrondie, recourbée; panse bilobée, moulurée; pied haut, oblique; fond creusé; couleur noire (7,5YR N2,5/); pâte noire, à dégraissant sableux, bien cuite. Ce type est très souvent décoré de guillochis sur les moulures et le col.

Phases III, IV, V.

No 2 : vase bilobé de type IIa; lèvre arrondie, recourbée se terminant en corniche; panse bilobée; pied haut; fond concave; couleur gris foncé $(2,5 \mathrm{YR}$ N3/) ; pâte blanche, à dégraissant sableux, bien cuite.

Macellum, phase $\mathrm{V}$.

No 3 : vase à lèvre fine; panse aplatie; pied haut, arrondi à la base; fond creusé ; couleur grise (10YR 4/1); pâte grise, à dégraissant sableux, bien cuite. La panse est ornée de guillochis en bandes régulières.

Puits daté du troisième quart du Ive $s$.

No 4 : vase à lèvre rentrante; panse ronde; cannelée; couleur noire $(7,5 \mathrm{YR}$ N2,5/); pâte grise, à dégraissant sableux, bien cuite. Il s'agit en fait d'une forme de dolium, réalisée en petite taille.

Sanctuaire germanique.

No 5: vase en lerra nigra; lèvre arrondie; col oblique, légèrement concave; panse arrondie; pied droit; fond creusé ; couleur noire $(2,5 Y$ N2,5); pâte grise, à dégraissant sableux, bien cuite.

Phase IV.

No 6 : vase à lèvre épaisse, rentrante, concave à l'extérieur; col oblique mouluré; couleur noire $(2,5 \mathrm{YR} \mathrm{N} 2,5 /)$; pâte gris clair, à dégraissant sableux, bien cuite. Type connu dans les nécropoles de la forêt de Compiègne, Champlieu et Mont-Chyprès.

Phase V.

No 7 : vase en terra nigra; lèvre fine, suivie d'un col concave; panse ronde, précédée d'une rainure; couleur noire ( $2,5 \mathrm{YR} \mathrm{N} 2,5 /)$; pàte gris clair, à dégraissant sableux. La lèvre et le col sont décorés de bandes lissées parallèles. Dérivé du type 341 de Chenet.

\section{Phase IV.}

No 8 : vase à lèvre épaisse suivie d'un col oblique; panse ronde, carénée; couleur noire $(2,5 \mathrm{YR} N 2,5)$; pâte grise, à dégraissant sableux, bien cuite. Le col et le haut de la panse sont décorés de bandes lissées parallèles. Type 342 de Chenet.

Phase V. 
No 9: vase à lèvre fine, arrondie, prolongée par un col oblique, mouluré ; couleur gris brun (5YR 3/1); pâte grise, à dégraissant sableux, bien cuite. Le col porte un décor de petites incisions ovalaires.

Phase IV.

No 10 : vase à lèvre épaisse, aplalie au summel; col concave; couleur grise (2,5YR N4/, N5/); pâte grise, à dégraissant sableux, bien cuite. Le col est décoré motifs lissés.

Phase IV.

No 11 : bouteille à lèvre anguleuse, en crochet; col oblique; panse ronde; pied arrondi; fond concave; couleur grise (10YR 5/1); pâte grise, à dégraissant sableux, bien cuite. Le col et le haut de la panse sont décorés de séries de bandes lissées parallèles.

Phases II, III, IV.

No 12 : bouteille en terra nigra; lèvre fine, arrondie; col oblique, légèrement concave; panse ronde; petit pied arrondi; fond creusé; couleur noire $(7,5 \mathrm{YR}$ N3/); pâte grise, à dégraissant sableux, bien cuite.

Phase IV.

No 13: cruche à bec tréflé; col concave; panse ronde; pied oblique, haut; fond concave, renflé au centre; couleur jaune (2,5Y 7/4) ; pâte jaune, à dégraissant sableux, bien cuite.

Phases II, III, IV, V.

\section{LA CÉRAMIQUE PEINTE (FIG. 8)}

No 1 : bol à lèvre allongée, arrondie; panse ronde; couleur jaune (5YR 6/6); pâte jaune. Le rebord est décoré d'un motif peint orange flammé.

La forme se rapproche de celles connues en Angleterre à Southwark (Marsh, 1978).

Phase I.

No 2: bol à lèvre droite, suivie d'une cannelure; couleur jaune (5YR 6/6); pâte jaune. Le haut est décoré de motifs orangés peints de lignes entrecroisées.

Phase I.

\section{LA CÉRAMIQUE GRANULEUSE (FIG. 9)}

No 1 : bol de type Alzei 29 , lèvre épaisse et grossièrement arrondie; paroi oblique; couleur grise (10YR $5 / 2)$; pâte grise, à dégraissant grossier.

Phase V.

Nos 2 à 5 : vases de type Alzei 27 , lèvres rentrantes, concaves au sommet; panses évasées; couleur grise à noire (10YR 6/1, 7,5Y $\mathrm{Y}$ N3/); pâtes grises à gros dégraissant.

Phases III, IV, V.
No 6 : vase de type Alzei 30, lèvre droite; couleur grise (10YR $5 / 2)$; pâte à gros dégraissant.

Phase V.

\section{LA CÉRAMIQUE NON TOURNÉE (FIG. 10)}

Nos 1 et 2 : assiettes non tournćcs à paroi ronde ou légèrement anguleuse; fonds plats; couleur noire (5YR 3/1, 7,5YR N3/, N2, 5/); pâte à dégraissant sableux; surfaces lissées.

Trouvées dans le secteur de la fosse-dépotoir de boucherie, contemporaine du sanctuaire germanique.

Forme connue à Erin (Beck ed., 1970, fig. 29, $\mathrm{n}^{\text {os }} 32$ à 34), Bochum Harpen (Beck ed., 1970, fig. 43, $n^{\circ} 8$ ), à Wijster (Van Es, 1967, type VC, fig. 148).

No 3 : bol non tourné ; lèvre arrondie; col concave; panse carénée; couleur noire $(7,5 \mathrm{YR}$ N2,5/); pâte à dégraissant sableux, bien cuite.

Phase IV. Forme connuc à Wijster (Van Es, 1967, type IC, fig. 100).

No 4 : bol non tourné; lèvre travaillée en festons; profil caréné; couleur gris foncé $(2,5 \mathrm{YR} \mathrm{N} 3 /)$; pâte grise à gros dégraissant. La panse est décorée d'incisions ovalaires.

Sanctuaire germanique.

Nos 5 et 6 : vases non tournés; lèvres arrondies, suivies d'un col concave; panse ovoïde ou ronde; couleur brun à noir $(2,5 \mathrm{YR}$ N2,5/, 5YR 2,5/1); pâtes à dégraissant sableux, bien cuites.

Sanctuaire germanique, puits G18. Des formes identiques ont été retrouvées dans le Pas-de-Calais à Labuissière et à Yzel-les-Hameaux, associées à du mobilier tardif. Ces types existent à Wijster (Van Es, 1967, type IVA, fig. 130), à Erin (Beck ed., 1970, fig. $30, \mathrm{n}^{\mathrm{os}} 1,2$ ), à Bochum-Harpen (Beck ed., 1970, fig. $\left.43, \mathrm{n}^{\circ} 7\right)$.

No 7 : tesson non tourné, décoré de bâtonnets et de cercles; couleur grise (10YR 4/1).

Phase IV. Ce décor est également connu à Wijster (Van Es, 1967, fig. 97, n"s 3 à 5) et à Erin (Van Es, 1967 , fig. $36, \mathrm{n}^{\mathrm{os}} 14$ à 17 ).

\section{LA RÉUTILISATION DE LA CÉRAMIQUE (FIG. 11)}

Cet ensemble provient du puits G.18, dont le comblement intervient dans le troisième quart du

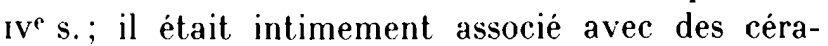
miques de type tardif, correspondant à la datation du puits.

No 1 : bol à collerette; lèvre fine, suivie d'une large collerette brisée; panse ronde; fond concave; couleur orange $(10 \mathrm{YR} 5 / 4)$; pâte orange, surface dorée au mica. 
Nos 2 et 3 : vases à panse ronde; lèvre fine; col concave très court: panse ronde cannelée; pied arrondi, oblique, ou en corniche ; fonds creusés ; couleur jaune orangé (10YR $6 / 4)$; pâte orange, à dégraissant sableux, bien cuite; surface dorée au mica. la panse du vase $n^{\circ} 2$ est décorée de bossettes. Le fond du vase $n^{\circ} 3$ est percé en passoire.

No 4 : vase caréné de type IIa en terra nigra; long col concave; panse carénée; pied oblique, arrondi; fond creusé, marqué d'un anneau saillant; couleur noire $(2,5 \mathrm{YR} \quad \mathrm{N} 2,5 /)$; pâte grise, à dégraissant sableux, bien cuite. La base de la panse est décorée de bandes lissées parallèles.

No 5 : vase tronconique de type IIIa; lèvre arrondie ; col tronconique; panse carénée; fond concave; couleur gris foncé $(7,5 \mathrm{YR} \mathrm{N} 3 /)$; pâte grise, à dégraissant sableux, bien cuite. Le col est décoré de bandes lissées parallèles; le haut de la panse d'incisions arrondies.

No 6: vase tronconique de type IIa, proche du précédent, mais réalisé en terra nigra; couleur noire $(2,5 Y R, N 2,5 /)$; pâte grise, à dégraissant sableux, bien cuite.

Cet ensemble est constitué de formes bien connues dans le Pas-de-Calais, notamment dans la nécropole de Baralle, et caractéristiques surtout de la première moitié du $I^{\mathrm{e}} \mathrm{s}$.

\section{LA CÉRAMIQUE DU SANCTUAIRE GERMANIQUE (FIG. 12)}

Les structures d'où proviennent ces céramiques ont déjà été publièes (Jacques, Tuffreau-Libre, 1991). Nous ne les décrirons donc pas.

\section{Fosse 30}

№ 1 : dolium; pied arrondi ; fond plat; panse ronde ; couleur brun clair $(7,5$ YR $5 / 2)$; pâte épaisse, brune, à dégraissant sableux, bien cuite. La partie supérieure manque.

No 2 : vase bilobé en terra nigra de type IIc; panse mouluréc; pied oblique, arrondi à la base; fond concave; couleur noire (2,5YR N3/); pâte à noyau noir, bords gris clair, dégraissant sableux, bien cuite. Les moulures sont décorées de guillochis réguliers.

No 3 : bol caréné de type XIc; lèvre arrondie, allongée; col bombé panse carénée; fond concave; couleur grise $(7,5 Y \mathrm{R} \times \mathrm{N} 3 /)$; pâte grise, à dégraissant sableux. bien cuite. Le col et l'intérieur du bol sont décorés de bandes lissées parallèles.

No 4 : cruche : col large : panse ronde; pied oblique; fond très légèrement creusé ; deux anses arrondies; couleur orange $(2,5 \mathrm{YR} 5 / 6)$; pâte blanchâtre. Le col et le haut de la panse sont décorés de bandes lissées irrégulières.

No 5 : cruche à lèvre cannelée; col droit; panse ronde moulurée; pied arrondi; fond creusé; anse cannelée; couleur jaune (2,כY 8/4); pâte jaune, à dégraissant sableux, bien cuite.

No $6:$ cruche à panse ronde; pied arrondi; fond creusé. Le col est absent; couleur grise (10YR 5/1, 4/1) pâte grise, à dégraissant sableux, bien cuite.

No 7 : bol à collerette très basse; fond creusé; couleur gris clair $(2,5 \mathrm{YN} \check{\partial} /)$; pâte grise, à dégraissant sableux, bien cuite. La collerette est ornée de bandes lissées.

No 8: couvercle; rebord arrondi; corps oblique; couleur grise (10YR 4/1); pâte grise, à dégraissant sableux, bien cuite.

No 9: bol rond de type Ia; lèvre arrondie, rentrante; panse ronde ; petit pied arrondi ; fond creusé ; couleur grise (10YR $4 / 1)$; pâte grise, à dégraissant sableux, bien cuite. L'intérieur du bol est décoré de bandes lissées parallèles.

No 10 : vase à panse aplatie de type $\mathrm{Ib}$; lèvre en léger crochet; panse aplatie, moulurée; pied oblique ; fond creusé ; couleur grise (2,5YR N4/); pâte grise, à dégraissant sableux, bien cuite.

\section{LA CÉRAMIQUE DU SANCTUAIRE GERMANIQUE (FIG. 13)}

\section{Fosse 20}

No 1 : bol caréné de type XIc; lèvre en crochet; col bombé; panse carénée; petit pied arrondi; fond concave; couleur grise (10YR 4/1); pâte grise, à dégraissant sableux, bien cuite.

№ 2: bol rond de type Ia; lèvre arrondie; panse ronde: fond légèrement creusé couleur grise $(7,5 \mathrm{YR}$ $\mathrm{N6/)}$; pâte grise, à dégraissant sableux, bien cuite.

No 3 : vase de type bilobé brisé; panse moulurée; fond concave; couleur grise (7,5YR N6/); pàte grise, à dégraissant sableux, bien cuite.

\section{Fosse 32}

No 4: bol rond de type Ia; lèvre arrondie; panse ronde: couleur grise $(2,5 \mathrm{YR} \mathrm{N} 3 /)$; pâte grise, à dégraissant sableux, bien cuite.

No 5: partie inférieure de vase ou cruche: panse légèrement aplatie; pied arrondi; fond creusé au centre; couleur grise $(7, \bar{Y} \mathrm{YR} \mathrm{N} 6 /)$; pâte grise à dégraissant sableux, bien cuite. La panse est décorée de bandes lissées parallèles. 
No 6: cruche au col brisé; panse ronde; pied oblique; fond presque plat; couleur orangée $(2,5 \mathrm{YR}$ 6/6); pâte blanche, à dégraissant sableux, bien cuite. Le col et la panse sont décorés de bandes lissées parallèles horizontales et verticales.

\section{Aire d'offrande}

No 7 : assiette à paroi oblique de type IIIa; lèvre s'affinant à l'extrémité; paroi oblique; petit pied droit ; fond plat ; couleur beige ; enduit rouge à l'intérieur et sur la lèvre (10R 4/8); pâte beige, à dégraissant sableux, bien cuite.

No 8 : bol à collerette; lèvre arrondie, suivie d'une cannelure; panse ronde, ornée au centre d'une colleretle brisée; pied šévasant; fond creusé, travaillé; couleur rouge $(10 \mathrm{R} 5 / 4,4 / 4)$; pâte grise, à dégraissant sableux, bien cuite.

\section{BIBLIOGRAPHIE}

\section{Bayard D.}

1980: La commercialisation de la céramique commune à Amiens, du milieu du $\mathrm{I}^{*}$ siècle à la fin du $\mathrm{II}^{*}$ siècle, Cahiers Archéologiques de Picardie, 7, p. 147-211, 27 fig.

Bayard D., Fournier J.

1978: In dépotoir du $\mathrm{w}^{\mathrm{r}}$ siècle rue Blaise-Pascal à Amiens. Cahiers Archéologiques de Picardie, 5, p. 191-199, 6 fig.

Beck H. ed.

1970 : Spätkaiserzeitliche Funde in Westfalen, Boden-allerlümer Westfalens, 12, 145 p., 53 fig.

Blondiaux J.

1990 : Les ossements humains du IV siècle de la rue Baudimont à Arras, in : Les culles à Arras au Bas-Empire, p. 94-95

Caron B., Monchy E.

1990 : Un ensemble céramique du IV" siècle à Courrières (Pasde-Calais), Gauheria, 22, p. 3-7, 2 fig.

Chenet G.

1941 : La cerramique d'Argonne du $I^{\circ}$ siecle, Fouilles et Documents d'Archéologie en France, 191 p., 38 fig.

De Laet S.J., Van Doorselaer A., Spitaels P., Thoen H. 1972: La nécropole gallo-romaine de Blicquy (Hainaut, Belgique), Dissertationes Archaeologicae Gandenses, 159 p., 152 fig.

Demolon P., Tuffreau-Libre M.

1976: Le puits gallo-romain $\mathrm{n}^{\circ} 37$ à Dourges, Gallia, 34, p. 285-292, 8 fig.

Giganon D., Tuffreau-Libre M.

1981 : Le site gallo-romain et mérovingien des Marnières a Saclay (Essonne), Bulletin du Vexin Français, p. 109-125. 11 fig.

\section{Gricourt D.}

1987 : Note préliminaire sur les monnaies de fouilles trouvées dans les niveaux théodosiens du quartier Baudimont à Arras
(Pas-de-Calais) : vers une réinterprétation de la problématique régionale, Cahiers Vumismatiques, 94, p. 331-338

Jacques A., Feugère M., Gricourt D., Tuffreau-Libre M.

1983 : Fouilles de sauvetage avant la construction de l'llòtel du Département, période gallo-romaine, Bulletin de la Commission d'Histoire el d'Archéologie du Pas-de-Calais, XI, 2, p. 259286, 24 tig.

Jacques A., Tuffreau-Libre $M$.

1978: Fosses gallo-romaines à Acq (Pas-de-Calais), Revue du Nord, LX, p. 865-872, 4 fig.

1991: L'occupation germanique sur le site gallo-romain d'Arras (Pas-de-Calais), Archäologisches Korrespondenzblatl, 21 , p. $409-419$.

\section{Jelski G.}

1980 : Les niveaux antiques et la céramique du chantier du Commissariat central d'Arras, Revue du Nord, 247, p. 833-859, 9 fig.

\section{Jobelot N.}

1986: La céramique fine et commune du balneaire de $W_{y}$ et essais de classification, Bulletin Archéologique du Vexin Français, p. 71-84, 11 fig.

\section{Lcclaire A., Tuffreau-Libre M.}

1991: Analyse d'un ensemble de céramiques gallo-romaines: la terra nigra tardive, in : La céramique du Bas-Empire en Gaule Belgique et dans les régions voisines, p. 51-52.

\section{Les cultes à Arras au Bas-Empire}

1990: Les culles à Arras au Bas-Empire, catalogue d'exposition, $120 \mathrm{p}$.

Machut B., Tuffreau-Libre $M$.

1991 : La cave gallo-romaine de (iraincourt-les-llavrincourt (Nord), Revue du Nord, 292, p. 159-172.

\section{Manière G.}

1966: Un puits funèraire de la fin du $\mathrm{I}^{\text {tr }}$ siècle aux Aquae Siccae (Cazeres, Haute-(iaronne), Gallia, XXIV, p. 101-160. 
Marsh G.

1978 : Early Second Century Fine Wares in the London Area, BAR, 57, p. 119-225, 25 fig.

Mertens J., Van Impe L.

1971 : Ilet laat gallo-romeins grafveld van Oudenburg, Archaeologia Belgica, 135, 275 p.; 92 pl. h.-t.

\section{Monchy E.}

1977 : Sépultures du Ier siècle ap. J.-C. à Vimy, 71 p., 30 fig.

Petit M.

1975: La céramique de type Mayen en région parisienne, Bulletin du Groupement Archéologique de Seine-et-Marne, 16, p. 99-109.

\section{Piton D., Marchand $\mathrm{H}$.}

1978 : Une nécropole du Ive siècle à Noyelles-sur-Mer, Cahiers Archéologiques de Picardie, 5, p. 199-230, 12 fig.

\section{Tuffreau-Libre M.}

1977 : La céramique commune gallo-romaine de la Forêt de Compiègne (Oise), au Musée des Antiquités .Nationales, Cahiers Archéologiques de Picardie, 4, p. 125-150, 19 fig.

1978 : I a céramique gallo-romaine dorée au mica dans le Nord de la France (.Nord et Picardie), Helinium, XVIII, p. 105-125, 14 fig.

1980a : La ceramique commune gallo-romaine dans le Nord de la France (Nord el Pas-de-Calais), Presses Universitaires de Lille, 287 p., 123 fig.

1980b: Un four gallo-romain à Labuissière (Pas-de-Calais), Gallia, 38, p. 293-309, 15 fig.
1988 : Les faciès régionaux de la céramique gallo-romaine du Nord de la France et du Bassin parisien, Helinium, XXVIII, p. 81-112, 15 fig.

1989 : La céramique gallo-romaine de Baralle, in : La nécropole à incinérations de Baralle (Pas-de-Calais), . Nord-Ouest Archéologie, 2, p. 205-223, 13 fig.

Tuffreau-Libre M., Jacques A.

1985 : La céramique gallo-romaine du $\mathrm{r}^{\mathrm{er}}$ siècle dans le Sud de l'Atrébatie, Gallia, 43, p. 127-145, 14 fig.

\section{Unverzagt $\mathbf{W}$.}

1976 : Die Keramik des Kastells Alzei, Römisch-germanische Kommission des deutschen archäologischen Instituts zu Frankfurt, 36 p., 3 pl. h.-t.

\section{Vanbrugghe $\mathrm{N}$.}

1989 : Recherches archéologiques à Famars (1986-1988), Revue du Nord, LXXI, 280, p. 79-103.

\section{Van Doorselaer A.}

1967 : Les nécropoles d'époque romaine en Gaule septentrionale, Dissertationes Archaeologicae Gandenses, X, 329 p.

Van Es W. A.

1967 : Wijster. A Vative Village beyond the Imperial Frontier, 150-425 A.D., $595 \mathrm{p}$.

Van Impe L.

1983 : Heit oudheidkundig bodemonderzoek in Donk (Germ.Ilerk-de-Stad) 1977-1982, in : Miscellanea Archeologica in honorem H. Roosens, Archaeologia Belgica, 255, Bruxelles, p. $65-94$. 